\title{
Derivation of a slow phase model of vortex-induced vibrations for smooth and turbulent oncoming flows
}

\author{
Vincent Denoël \\ Structural \& Stochastic Dynamics, University of Liège, Liège, Belgium
}

\section{A R T I C L E I N F O}

\section{Article history:}

Received 24 February 2020

Received in revised form 15 July 2020

Accepted 4 September 2020

Available online $\mathrm{xxxx}$

\section{Keywords:}

Wake-Oscillator model

Facchinetti model

Synchronization

Stochastic Van der Pol oscillator

Lock-in

Turbulence

\begin{abstract}
A B S T R A C T
This paper analyzes the influence of turbulence on a wake-oscillator model. Turbulence is introduced by randomizing the model proposed by Facchinetti et al. under the quasisteady assumption. A multiple scale analysis of the deterministic model shows that the response is governed by a dimensionless group $\mathcal{D}$, expressed as a function of the amplitudes of the forcing terms in the two governing equations, the total (aerodynamic plus structural) damping and the parameter $\varepsilon$ of the fluid Van der Pol oscillator. The influence of turbulence is interpreted as a stochastic noise of small intensity and with a slower timescale than the (fast) oscillations, which is typical of wind engineering applications. A slow phase model of the problem is then derived by assuming that the small turbulence drives the system only slightly away from its limit cycle in smooth flow conditions. Standard modeling techniques borrowed from other fields of physics, in particular the observation of phase shifts and their accumulation, are used to highlight conditions under which the turbulence of the oncoming flow might reduce the amplitudes of vibrations of the body. The slow phase model is derived in smooth flow conditions, then extended to turbulent flow. It recalls that the phase plays a central role in synchronization problems, and that the response amplitude should only be considered as a sub-product of the slow phase. The slow phase model is expressed by means of a first order nonlinear differential equation for the phase and a memoryless transformation for the response amplitudes. Its solution is explicit and simple in some limiting cases. In particular, for small turbulence intensity, the response is shown to be insensitive to turbulence when its frequency content is not low enough. This major dependence upon the frequency content of the turbulence explains that the reduction of VIV due to turbulence cannot be explained by the turbulence intensity only, as usually considered today. The required relative smallnesses of the turbulence and its frequency content naturally appear in the derivation, which is led in a dimensionless manner. Finally, the present study constitutes an analysis of a phenomenological model which could be used in a much wider concept than of the elastically-mounted circular cylinder.
\end{abstract}

(c) 2020 Elsevier Ltd. All rights reserved.

\section{Introduction}

Models describing vortex-induced vibrations (VIV) of bodies immersed in smooth flows are based on an equation of motion for the structural vibrations which is appropriately modified or complemented in order to model the interaction with the fluid in a more or less sophisticated manner. Existing two-dimensional models are typically classified in three different families (Païdoussis et al., 2010). In family I (forced), the fluid is modeled as a passive external lift force on the

E-mail address: v.denoel@uliege.be. 


\section{ARTICLE IN PRIESS}

structural oscillator; the root-mean-square value of the lift is calibrated with ad hoc measurement (Simiu and Scanlan, 1996). In family II (forced feedback), the lift force depends on the state-usually the amplitude- of the structural oscillator. The model consists therefore in a nonlinear differential equation which is capable of modeling the self-limiting features of this problem (e.g. Larsen, 1995; Lupi et al., 2018; Marra et al., 2011). In family III (wake-oscillator), the fluid is more accurately modeled as a dynamical system with a limit cycle. A Van der Pol or Rayleigh differential equation is typically used for this and is coupled with the structural oscillator in various possible ways (Hartlen and Currie, 1970; TAMURA and Matsui, 1980; Krenk and Nielsen, 1999; Facchinetti et al., 2004; Farshidianfar and Zanganeh, 2010). After an exhaustive analysis, Facchinetti et al. (2004) suggested to adopt a forcing of the fluid equation which is proportional to the acceleration of the elastic body. Tamura's model (TAMURA and Matsui, 1980) is based on a formal derivation and physical arguments. By combining the velocity and acceleration of the body into the forcing term of the fluid, it is more general than Facchinetti's model. It is for instance able to mimic the asymmetry of the lock-in response, as in the seminal Birkhoff (1953) or Hartlen and Currie (1970) models.

The influence of turbulence on VIV of elastically mounted cylinders or more complicated shapes like bridge decks has been experimentally investigated in many studies (Howell and Novak, 1980; Matsumoto et al., 2001; So et al., 2008; Trush et al., 2017). It appears that there is no clear consensus on the influence of turbulence. Anyways, the answer shall not necessarily be dichotomic; at least, the influence of turbulence should depend on the type of turbulence (its frequency content) and its intensity. In some studies, turbulence is shown to have a strong effect on the synchronization mechanism and may lead to a complete suppression of VIV (Cao and Cao, 2017). Other works tend to indicate that the grid turbulence of the oncoming flow has virtually no influence on the response amplitude (Goswami et al., 1993). The divergent conclusions of these studies indicate the complexity of the problem. In fact, the flow physics in the whole lock-in regime is much more complex than the von Karman vortex street, which appears for fixed bluff bodies (Williamson and Roshko, 1988; Pasto, 2008). Computational fluid dynamics simulations have shed a different light on the problem and helped understand the peculiarities of the flow, locally, where detachment is affected by turbulence (Guilmineau and Queutey, 2004; Nguyen et al., 2018). Yet, the analysis that is carried out in this paper does not pretend to enter such a detailed level of analysis and, quite the opposite, aims at analyzing a simple and adjustable phenomenological model.

Available mathematical models to describe VIV in a turbulent oncoming flow are much scarcer than for smooth flows. Civil engineering applications such as bridge aerodynamics (Hansen, 2007; Komatsu and Kobayashi, 1980; Larsen, 1995; Sarwar and Ishihara, 2010; Wu and Kareem, 2013), cable aerodynamics (Dyrbye and Hansen, 1996; Matsumoto et al., 2001; Denoël and Andrianne, 2017), chimneys (Daly, 1986; Pritchard, 1984; Ruscheweyh and Sedlacek, 1988) and tall buildings (Kawai, 1992) are all concerned by vortex induced vibrations in the atmospheric boundary layer where the magnitude of the fluctuating component (turbulence) can reach up to $20 \%$ or more of the average wind velocity. There are therefore obvious needs for simple models of VIV in the presence of noise. In the family of externally forced models (family I), Vickery and coworkers (Vickery and Clark, 1972; Vickery and Basu, 1983; Vickery, 1995, 1998) have proposed a loading model of tapered stacks which is still widely used today. It constitutes a cornerstone of the modeling of the influence of turbulence on vortex-induced vibrations. This model has been constructed as a simple generalization of the externally forced model (family I), by modeling the aerodynamic loading as a narrow band stochastic process, instead of a deterministic harmonic loading. Stochastic versions of the family II models are also available in the literature, see e.g. Le and Caracoglia (2015). Following the same spirit, this paper presents and analyzes a randomized version of a simple wake oscillator model (family III). A seminal randomized version of the Hartlen-Currie model is studied in Benaroya and Lepore (1983) by means a convolution integral of the linear structural oscillator and the derivation of the FokkerPlanck-Kolmogorv equations for the nonlinear fluid equation, externally forced by a narrow-band stochastic structural motion. Using a Monte Carlo approach, Krenk's wake-oscillator model, based on an energy balance, has been studied in stochastic conditions using the quasi-steady approach too (Nielsen and Krenk, 1997), after which it is concluded that the stochastic response resembles the observed behavior in experiments and full-scale testing. Monte Carlo simulations are the simplest way to tackle non deterministic problems since the solution just requires the generation of synthetic realizations of the turbulent flow and the statistical analysis of the response (Tagliaferri and Srinil, 2017). This approach has also been used to determine the response of structures with many more degrees-of-freedom (Li, 2019) or in more complicated geometries than the 2-D case of simple wake-oscillator models (Ulveseter et al., 2017). Although being simple to implement, the Monte Carlo approach does not offer the same depth of understanding as explicit closed form solutions which are only valid in some simple configurations, e.g. in the analysis of the Van der Pol equation alone in stochastic loading conditions (Leung, 1995; Gu et al., 2011). More recently Facchinetti's wake-oscillator model has also been studied in stochastic conditions, under the quasi-steady assumption. While Aswathy and Sarkar study the stochastic version of this model and the influence of parametric noise with the help of Monte Carlo simulations (Aswathy and Sarkar, 2019), Shoshani examines the responses of the coupled system by means of stochastic averaging (Shoshani, 2018).

As explained above the quasi-steady assumption shall not strictly apply, nor be used in a predictive manner (a simple argument is to observe that both the Strouhal number and the aerodynamic coefficients are affected by turbulence of the oncoming flow), it is still interesting to understand the specific features of a model. This paper does not pretend to offer more that the understanding of a phenomenological model, that could be fitted to observed experimental or in-situ measured data. With this in mind, this paper analyzes the stochastic version of the Facchinetti model with the pragmatical tools of nonlinear stochastic dynamics. Quite interestingly, it also introduces a slow phase model for this problem. As seen next, this model is simple enough to derive approximate solutions of the problem, encapsulating the major problem 
parameters in the governing leading dimensionless groups. This approach might be attractive to complement attempts at standardizing the design of VIV-sensitive structures (Hansen, 2007; Standard, 1991).

The paper is organized as follows. In Section 2, the considered (Facchinetti) model is introduced, together with its randomized version. The averaged version of the deterministic system is introduced in Section 3. This derivation emphasizes the central role of the dimensionless group $\mathcal{D}$ and the slow phase between the structural and fluid oscillators. It is also emphasized that it is not necessary to resolve the fast dynamics as long as the slow dynamics of the phase and the response envelopes are studied. In Section 4, the stochastic version of the problem, is presented. Phase slips and their accumulation are evoked to explain why and how turbulence is able to affect the VIV response. Then, a slow phase model is derived and simple analytical solutions are provided. They offer a simple outlook on the parameters and dimensionless groups driving the response of the coupled system in stochastic conditions. Illustrations are finally given to feed the discussion about the validity of the proposed simplified solutions.

\section{Nomenclature}

1

2

3

4

5

6

7

8

9

10

\begin{tabular}{|c|c|c|c|c|c|}
\hline Model parameters & Units & & & Scaling & Dimensionless \\
\hline$t$ & $\mathrm{~T}, \mathrm{~T}^{-1}$ & & Time & $t^{\star}=1 / \omega_{0}$ & $\tau$ \\
\hline$y(t)$ & $\mathrm{L}$ & & Transverse displacement of cylinder & $y^{\star}=\varepsilon D$ & $\mathcal{Y}(\tau, T=\varepsilon \tau)$ \\
\hline$q(t)$ & - & & Generalized lift (Fdlb model) & $q^{\star}=1$ & $\mathcal{Q}(\tau, T=\varepsilon \tau)$ \\
\hline$\varepsilon$ & - & a & van der Pol coefficient in wake equation & & \\
\hline$D$ & $\mathrm{~L}$ & & Crossflow dimension of cylinder & & \\
\hline$\omega_{0}$ & $T^{-1}$ & & $\begin{array}{l}\text { Natural circular frequency of elastically } \\
\text { mounted cylinder }\end{array}$ & & \\
\hline$m_{\mathrm{S}}, m_{\mathrm{F}}, c_{\mathrm{S}}, k_{\mathrm{S}}$ & mixed & & $\begin{array}{l}\text { Parameters of the Facchinetti-de } \\
\text { Langre-Biolley... }\end{array}$ & & \\
\hline$\rho, U_{\infty}, C_{D}, C_{L}^{0}, \mathcal{A}_{0}$ & mixed & & ...model (Fdlb), see definition in (1) & & \\
\hline$\mu$ & - & & $\begin{array}{l}\text { Fluid/Structure mass ratio } \\
\left(\mu=\frac{\rho \pi D^{2}}{4\left(m_{\mathrm{S}}+m_{\mathrm{F}}\right)}=\frac{\rho}{\rho_{\mathrm{S}}}\right) \text {, see }(4)\end{array}$ & & $\mathcal{M}_{0}$ \\
\hline St & - & & Strouhal number & & \\
\hline$f_{\text {shedding }}$ & $T^{-1}$ & & Shedding frequency $\left(f_{\text {shedding }}=\operatorname{St} U_{\infty} / D\right)$ & & \\
\hline $\mathrm{Sc}^{\star}=\mathrm{Sc} \xi / \xi_{S}$ & - & & $\begin{array}{l}\text { Scruton number, defined with total } \\
\text { damping, see (17) }\end{array}$ & & \\
\hline $\mathrm{SG}^{\star}=\mathrm{SG} \xi / \xi_{s}$ & - & & $\begin{array}{l}\text { Skop-Griffin number, defined with total } \\
\text { damping, see (18) }\end{array}$ & & \\
\hline$u(\tau)$ & $\mathrm{L} \mathrm{T}^{-1}$ & & $\begin{array}{l}\text { Turbulence velocity (Gaussian random } \\
\text { process) }\end{array}$ & $\sigma_{u}$ & $\mathcal{U}(T=\varepsilon \tau)$ \\
\hline$\sigma_{u}$ & $\mathrm{~L} \mathrm{~T}^{-1}$ & & Standard deviation of turbulence velocity & & \\
\hline$I_{u}$ & & a & Turbulence intensity $\left(I_{u}=\sigma_{u} / U_{\infty}\right)$ & & \\
\hline$S_{u}$ & $\mathrm{~L}^{2} \mathrm{~T}^{-1}$ & & Power spectral density of turbulence & $\sigma_{u}^{2} / \omega_{0}$ & $S_{\mathcal{U}}\left(\tilde{\omega}=\omega / \omega_{0}\right)$ \\
\hline$\alpha$ & - & a & $\begin{array}{l}\text { Dimensionless characteristic turbulence } \\
\text { frequency }\end{array}$ & & \\
\hline
\end{tabular}

\begin{tabular}{lll}
\hline \hline Model parameters & Units & \\
\hline $\mathcal{M}_{0}$ & - & \\
$\xi_{s}, \xi_{a}$ & - & a \\
$\xi=\xi_{s}+\xi_{a}$ & - & a \\
$\Omega$ & - & b \\
$\delta=(\Omega-1) / \xi$ & - & \\
$\mathcal{D}$ & -
\end{tabular}

\begin{tabular}{l}
\hline$R_{y}(T)$ \\
$R_{q}(T)$ \\
$\psi(T)$ \\
$\xi_{0}=\xi / \varepsilon$ \\
$\mathcal{I}_{0}=I_{u} / \varepsilon$ \\
$\Upsilon(T)=\cot \psi(T)$ \\
$\Upsilon_{\mathrm{LC}}=\cot \psi_{\mathrm{LC}}$ \\
$\mathcal{B}$ \\
$\mathcal{V}$
\end{tabular}

Dimensionless fluid/structure mass ratio, see (10)

Structure and fluid damping ratios, see (4)

Total damping ratio, see (4)

Mistuning, bifurcation parameter, see (4)

Centered and scaled mistuning, bifurcation parameter, see (22)

Unique dimensionless group characterizing the deterministic response of the Fdlb model, see (28)

Slow envelope of the structural response $\mathcal{Y}$

Slow envelope of the reduced lift $\mathcal{Q}$

Slow phase between $\mathcal{Y}(\tau, T)$ and $\mathcal{Q}(\tau, T)$

Reduced damping ratio

Reduced turbulence intensity

Cotangent of the slow phase, see (45)

Cotangent of the slow phase on the limit cycle, $\Upsilon_{\mathrm{LC}} \equiv \Upsilon_{\mathrm{LC}}(\delta ; \mathcal{D})$, see $(27)$

Dimensionless group characterizing the stochastic response of the Fdlb model, see (47)

VIV reduction factor accounting for the frequency content of turbulence (vs. the slow memory of the structure), see (54) 


\begin{tabular}{lll}
\hline Model parameters & Units & \\
\hline$\Delta \Upsilon(T)$ & - & Deviation from limit cycle solution $\Delta \Upsilon(T)=\Upsilon(T)-\Upsilon_{\mathrm{LC}}$ \\
$\mu_{\Delta \Upsilon}, \sigma_{\Delta \Upsilon}$ & - & Average and standard deviation of the deviation from limit cycle solution \\
$\mu_{\Upsilon}, \sigma_{\Upsilon}$ & - & Average and standard deviation of the cotangent of the slow phase, \\
$r_{\Upsilon}$ & - & Reduction factor of the maximum structural response, random variable \\
\hline
\end{tabular}

Symbols with a naught underscore ${ }_{0}$ indicate quantities that have been scaled by a power of $\varepsilon\left(\xi_{0}, \mathcal{I}_{0}, \mathcal{A}_{0}, \mathcal{M}_{0}\right)$. Calligraphic symbols are used to represent dimensionless numbers or groups.

a Small numbers.

\section{Considered wake-oscillator model}

The Facchinetti-de Langre-Biolley (Fdlb) model reads (Facchinetti et al., 2004)

$$
\begin{aligned}
\left(m_{\mathrm{S}}+m_{\mathrm{F}}\right) \ddot{y}+\left(c_{S}+\frac{C_{D}}{2} \rho U_{\infty} D\right) \dot{y}+k_{S} y & =\frac{1}{4} \rho U_{\infty}^{2} D C_{L}^{0} q \\
\ddot{q}+2 \pi \mathrm{St} \frac{U_{\infty}}{D} \varepsilon\left(q^{2}-1\right) \dot{q}+\left(2 \pi \mathrm{St} \frac{U_{\infty}}{D}\right)^{2} q & =2 \mathcal{A}_{0} \frac{\ddot{y}}{D}
\end{aligned}
$$

where $y(t)$ (units: L) and $q(t)$ (units: -) represent the two degrees-of-freedom associated with the cross-flow structural motion and the lift force resulting from vortex shedding. The parameters of the model are $m_{\mathrm{S}}, c_{\mathrm{S}}$ and $k_{\mathrm{S}}$, the mass, viscosity and stiffness of the structure (per unit length), $D$ the cylinder diameter (or characteristic cross-flow dimension), $\rho$ and $U_{\infty}$ the density and the constant velocity of the fluid, $C_{D}$ and $C_{L}^{0}$ the stationary drag and the magnitude of the lift fluctuations on the fixed body and St the Strouhal number. Finally $\mathcal{A}_{0}$ is a dimensionless parameter related to the influence of the structural motion on the dynamics of the wake $\left(2 \mathcal{A}_{0}\right.$ corresponds to symbol $A$ used in Facchinetti et al., 2004), while $\varepsilon$ is another dimensionless parameter that describes the memory in the wake equation and is related to the magnitude of the nonlinearity in the Van der Pol equation for the wake, therefore to the strength of the limit cycle. The equivalent mass of displaced fluid $m_{\mathrm{F}}=C_{M} \rho D^{2} \frac{\pi}{4}$ (which is negligible in wind engineering applications) is added to the structural mass $m_{\mathrm{S}}$ in order to define the structural circular frequency $\omega_{0}=\sqrt{k_{\mathrm{S}} /\left(m_{\mathrm{S}}+m_{\mathrm{F}}\right)}=\sqrt{k_{\mathrm{S}} / m}$. The Fdlb model has been imagined for subcritical flows $300 \lesssim \operatorname{Re} \lesssim 1.5 \cdot 10^{5}$ (Facchinetti et al., 2004). Addition of turbulence in the oncoming flow is known to promote early transition to supercritical (Blackburn and Melbourne, 1996), which truncates the domain of applicability of this model.

\subsection{Dimensionless version of problem}

A dimensionless version of the governing equations is obtained by introducing the characteristic time $t^{\star}=1 / \omega_{0}$ and a characteristic structural displacement $y^{\star}$ (to be defined soon) and defining

$$
\tau=\frac{t}{t^{\star}} \quad, \quad \mathcal{Y}(\tau)=\frac{y[t(\tau)]}{y^{\star}} \quad \text { and } \quad \mathcal{Q}(\tau)=\frac{q[t(\tau)]}{q^{\star}}
$$

with $q^{\star}$ arbitrarily chosen as 1 . With the usual dimensionless numbers, characterizing the structural damping ratio $\xi_{s}$ and aerodynamic damping ratio $\xi_{a}$, as well as the reduced wind velocity $\Omega$ and the mass ratio $\mu$

$$
\xi_{s}=\frac{c_{S}}{2 \sqrt{k_{S} m}}, \quad \xi_{a}=\frac{\rho U_{\infty} D}{4 \sqrt{k_{S} m}} C_{D}, \quad \Omega=\frac{\mathrm{St} U_{\infty} / D}{\omega_{0} / 2 \pi}=\frac{f_{\text {shedding }}}{f_{0}}, \quad \mu=\frac{\rho \pi D^{2}}{4 m}=\frac{\rho}{\rho_{\mathrm{S}}},
$$

with $\rho_{\mathrm{S}}$ the equivalent density of the cylinder, the governing equations become

$$
\begin{aligned}
\mathcal{Y}^{\prime \prime}+2\left(\xi_{s}+\xi_{a}\right) \mathcal{Y}^{\prime}+\mathcal{Y} & =\frac{1}{4} \frac{1}{k_{s} y^{\star}} \rho U_{\infty}^{2} D C_{L}^{0} \mathcal{Q}=\frac{\mu}{4 \pi^{3}} \frac{D}{y^{\star}}\left(\frac{\Omega}{\mathrm{St}}\right)^{2} C_{L}^{0} \mathcal{Q} \\
\mathcal{Q}^{\prime \prime}+\varepsilon \Omega\left(\mathcal{Q}^{2}-1\right) \mathcal{Q}^{\prime}+\Omega^{2} \mathcal{Q} & =2 \mathcal{A}_{0} \frac{y^{\star}}{D} \mathcal{Y}^{\prime \prime}
\end{aligned}
$$

where the prime symbol denotes derivatives with respect to the dimensionless time $\tau$. In the wake equation, the small parameter $\varepsilon$ plays a major role. It is small to moderate, say in the range [0.01; 0.4$]$; it is always certainly not equal to zero, otherwise there would not be any synchronization. It appears therefore as a good candidate to derive an asymptotic solution of this problem. In order to derive a consistent distinguished limit, we study the solutions of the problem where the structural displacement $y$ is small compared to the diameter $D$ of the body, which anyways correspond to the domain of applicability of the wake-oscillator model (2-S shedding mode, see e.g. Williamson and Roshko, 1988). It is therefore natural to choose

$$
y^{\star}=\varepsilon D
$$


as a characteristic displacement, and to solve the problem for $\mathcal{Y}(\tau) \sim 1$. Seeking applications in wind engineering, the (fluid-over-structure) mass ratio is assumed to be very small, of the order of $\varepsilon^{2}$ or smaller (typically $10^{-3}$ ). The mass ratio $\mu$ is therefore written $\mu=\mu_{0} \varepsilon^{2}$ with $\mu_{0} \sim 1$ so that the governing equations become

$$
\begin{aligned}
\mathcal{Y}^{\prime \prime}+2\left(\xi_{s}+\xi_{a}\right) \mathcal{Y}^{\prime}+\mathcal{Y} & =2 \varepsilon \mathcal{M}_{0} \Omega^{2} \mathcal{Q} \\
\mathcal{Q}^{\prime \prime}+\varepsilon \Omega\left(\mathcal{Q}^{2}-1\right) \mathcal{Q}^{\prime}+\Omega^{2} \mathcal{Q} & =2 \varepsilon \mathcal{A}_{0} \mathcal{Y}^{\prime \prime}
\end{aligned}
$$

where $\mathcal{M}_{0} \sim 1$ has been defined as

$$
\mathcal{M}_{0}:=\frac{\mu}{8 \pi^{3} \varepsilon^{2}} \frac{C_{L}^{0}}{S^{2}} .
$$

It therefore carries information about the mass ratio, the lift force and the shape of the body (through the Strouhal number and lift coefficient). The Skop-Griffin parameter is expressed as a function of $\mathcal{M}_{0}$ because it is the sole dimensionless group carrying information about the mass ratio. The smallness of the righthand side of (8) also translates that the lift created by vortex shedding is small compared to the elastic forces in the body (energy is slowly pumped in/out of the system).

In this paper, the turbulence of the oncoming flow is introduced in the model, following the quasi-steady approach (Simiu and Scanlan, 1996; Dyrbye and Hansen, 1996). A randomized version of the governing equations, obtained by substituting $U_{\infty}+u(t)$ for $U_{\infty}$ in (8)-(9) is here developed. It is assumed that $u(t)$ is a zero-mean Gaussian stochastic process with known variance $\sigma_{u}$ and known power spectral density $S_{u}$ (PSD). The validity is this randomization procedure is still debatable with regards to the available information today, although this is common practice as stated in the introduction; however, seeking applications in the atmospheric boundary layer, the turbulence of the oncoming flow is characterized by a slow timescale which is one or several orders of magnitude slower than the structural oscillations and, hence, aeroelastic effects. This argument contributes to the separation of the aeroelastic and buffeting effects, which is favorable to this decomposition, (see Cremona et al., 2002, p.88). Noticing that $U_{\infty}$ appears in the definition of $\xi_{a}$ and $\Omega$, this substitution readily provides the resulting system of equations in its most simple form,

$$
\begin{aligned}
\mathcal{Y}^{\prime \prime}+2\left(\xi_{s}+\xi_{a}\left(1+I_{u} \mathcal{U}\right)\right) \mathcal{Y}^{\prime}+\mathcal{Y} & =2 \varepsilon \mathcal{M}_{0}\left(1+I_{u} \mathcal{U}\right)^{2} \Omega^{2} \mathcal{Q} \\
\mathcal{Q}^{\prime \prime}+\varepsilon \Omega\left(1+I_{u} \mathcal{U}\right)\left(\mathcal{Q}^{2}-1\right) \mathcal{Q}^{\prime}+\Omega^{2}\left(1+I_{u} \mathcal{U}\right)^{2} \mathcal{Q} & =2 \varepsilon \mathcal{A}_{0} \mathcal{Y}^{\prime \prime}
\end{aligned}
$$

where a dimensionless turbulence $\mathcal{U}(\tau)$ has been defined by normalizing the velocity fluctuation $u(t)$ by is standard deviation $\sigma_{u}$,

$$
u[t(\tau)]=\sigma_{u} \mathcal{U}(\tau)=I_{u} U_{\infty} \mathcal{U}(\tau),
$$

with $I_{u}=\sigma_{u} / U_{\infty}$ the turbulence intensity, usually in the range [0;0.25]; it is therefore considered as a small number, i.e. $I_{u} \ll 1$.

The objective of the study reported in this paper is to analyze this set of equations with the qualitative and quantitative tools in nonlinear dynamics, in order to sketch the important features of this model and explain them with simple concepts. As seen next, this will provide a possible explanation of the influence of turbulence on the lock-in phenomenon. In Sections 3 and 4 we derive an asymptotic solution of this problem. In Section 5, we study the accuracy of the proposed model by comparison with Monte Carlo simulations of (11), which will serve as a reference solution.

\subsection{The dimensionless turbulence $\mathcal{U}(\tau)$}

In the governing equations, $\mathcal{U}(\tau)$ is a zero-mean unit-variance process indexed on the dimensionless time $\tau\left(\mu_{\mathcal{U}}=0\right.$, $\left.\sigma_{\mathcal{U}}=1\right)$. The corresponding PSD can be expressed as a function of the power spectral density of $u(t)$, since $\mathcal{U}(\tau)$ and $u(t)$ are related to each other by means of a stretch and a re-scaling. Let us write $S_{u}(\omega ; \mathbf{p})$ the PSD of $u(t)$, where $\mathbf{p}$ represents some possible parameters of the model such as $\sigma_{u}$, the variance of $u(t)$, or the turbulence lengthscale $L_{u}^{x}$, or the mean wind velocity $U_{\infty}$. For instance, the following PSD's

$$
S_{u}\left(\omega ; \frac{U}{L}, \sigma_{u}^{2}\right)=\sigma_{u}^{2} \frac{0.546 \frac{L}{U}}{\left(1+1.64 \frac{L}{U}|\omega|\right)^{5 / 3}} \quad, \quad S_{u}\left(\omega ; a, \sigma_{u}^{2}\right)=\sigma_{u}^{2} \frac{a}{\pi} \frac{1}{\omega^{2}+a^{2}},
$$

represent an existing model of the power spectral density of atmospheric turbulence (Dyrbye and Hansen, 1996) and the power spectral density of the Ornstein-Uhlenbeck process (Papoulis and Pillai, 2002). This latter example will be used in the following illustrations. The PSD of the zero-mean unit-variance process $\mathcal{U}(\tau)$ is then given by

$$
S_{\mathcal{U}}(\tilde{\omega} ; \alpha)=\frac{\omega_{0}}{\sigma_{u}^{2}} S_{u}\left(\tilde{\omega} \omega_{0} ; \alpha \omega_{0}, \sigma_{u}^{2}\right)
$$

where $\tilde{\omega}=\omega / \omega_{0}$ is the dimensionless frequency parameter (associated with time $\tau$ ) and $\alpha$ a dimensionless characteristic frequency of the turbulence velocity. For the two examples given above $\alpha=U / L \omega_{0}$ and $\alpha=a / \omega_{0}$, respectively. This number relates the characteristic frequency of turbulence $(U / L$ or $a)$ to the characteristic frequency $\omega_{0}$ of the structure. It is typically small in wind engineering applications, in the range $\left[10^{-3} ; 10^{-1}\right]$. Its smallness will also be exploited in the 
Table 1

Typical range of variation of the parameters of the problem.

\begin{tabular}{llllll}
\hline $\begin{array}{l}\text { Model } \\
\text { parameters }\end{array}$ & $\begin{array}{l}\text { Range of } \\
\text { variation }\end{array}$ & $\begin{array}{l}\text { Governing } \\
\text { parameters }\end{array}$ & $\begin{array}{l}\text { Range of } \\
\text { variation }\end{array}$ & $\begin{array}{l}\text { Reduced } \\
\text { parameters }\end{array}$ & $\begin{array}{l}\text { Range of } \\
\text { variation }\end{array}$ \\
\hline$\xi_{s}$ & {$\left[5 \cdot 10^{-4}, 10^{-2}\right]$} & $\Omega$ & {$[0.5,1.5]$} & $\mathcal{D}$ & {$[1 ; 30]$} \\
$\xi_{a}$ & {$\left[10^{-3}, 5 \cdot 10^{-2}\right]$} & $\varepsilon$ & {$[0.05 ; 0.3]$} & $\xi_{0}=\xi / \varepsilon$ & {$\left[5 \cdot 10^{-2} ; 1\right]$} \\
& & $\xi=\xi_{s}+\xi_{a}$ & {$\left[10^{-3}, 5 \cdot 10^{-2}\right]$} & $\mathcal{I}_{0}=I_{u} / \varepsilon$ & {$[0 ; 10]$} \\
Turbulence & & $\mathcal{M}_{0}$ & {$[0.01 ; 1]$} & & \\
$\alpha$ & {$\left[10^{-3} ; 10^{-1}\right]$} & $\mathcal{A}_{0}$ & {$[1 ; 10]$} & & \\
$I_{u}$ & {$[0 ; 0.25]$} & & & & \\
\hline
\end{tabular}

sequel in order to derive closed-form solutions of this problem. The PSDs of the normalized turbulence corresponding to (13) are therefore given by

$$
S_{\mathcal{U}}(\tilde{\omega} ; \alpha)=\frac{0.546}{\left(\alpha^{3 / 5}+1.64|\tilde{\omega}|\right)^{5 / 3}} \quad, \quad S_{\mathcal{U}}(\tilde{\omega} ; \alpha)=\frac{\alpha}{\pi} \frac{1}{\tilde{\omega}^{2}+\alpha^{2}} .
$$

The normalization property of the PSD translates into

$$
\int_{-\infty}^{+\infty} S_{\mathcal{U}}(\tilde{\omega} ; \alpha) d \tilde{\omega}=1
$$

regardless of the value of the parameter $\alpha$ (Denoël and Carassale, 2015). Notice that $S_{\mathcal{U}}(\tilde{\omega} ; \alpha)$ might be expressed as a function of additional parameters, should the original density $S_{u}(\omega)$ be more complex than (13). It is just independent of $\sigma_{u}$ as a result of the re-scaling.

\subsection{Order of magnitude of the parameters of the model}

In typical applications, $\xi_{s}, \xi_{a}, \varepsilon, \alpha$ and $I_{u}$ are small parameters. The orders of magnitude of the parameters of the model are given in Table 1 while the numerical values that are chosen in the following illustrations (Data Set 1 and Data Set 2) are given in Table 2. These values are inspired from the examples given in TAMURA and Matsui (1980) (Data set 1) and Facchinetti et al. (2004) (Data set 2). Some other important rescaled parameters such as $\xi_{0}=\xi / \varepsilon=\left(\xi_{s}+\xi_{a}\right) / \varepsilon$ and dimensionless groups such as $\mathcal{D}=\mathcal{A}_{0} \mathcal{M}_{0} / \xi_{0}^{2}$ will naturally appear in the following developments. In order to be exhaustive and collect all relevant information at the same place, the values for these dimensionless groups are also reported in the same Tables. The origin of these parameters will become clearer in Sections 3 and 4.

The parameters of the Fdlb model can be related to other standard mass-damping parameters that are used to describe vortex-induced vibrations. In particular, using the standard definition for the Scruton number (Scruton, 1981)

$$
\mathrm{Sc}^{\star}:=\frac{4 \pi\left(\xi_{s}+\xi_{a}\right) m}{\rho D^{2}}=\pi^{2} \frac{\xi}{\mu},
$$

but referring to the total damping $\xi$ (instead of only the structural damping $\xi_{s}$ ), with $\mu$ the mass ratio defined in (10) and $\xi=\xi_{s}+\xi_{a}$ the total damping, the Skop-Griffin parameter defined by $\mathrm{SG}^{\star}:=2 \pi \mathrm{St}^{2} \mathrm{Sc}^{\star}$ (Griffin and Koopmann, 1977) is found to be given by

$$
\mathrm{SG}^{\star}=2 \pi^{3} \mathrm{St}^{2} \frac{\xi}{\mu}=\frac{\xi C_{L}^{0}}{4 \varepsilon^{2} \mathcal{M}_{0}} .
$$

These notations are different from the usual mass-damping parameter SG which is calculated with the structural damping only, SG $=2 \pi^{3} \mathrm{St}^{2} \xi_{s} / \mu$, or from the usual Scruton number Sc $=\pi^{2} \xi_{s} / \mu$ also defined for the structural damping only. Rewriting the former equation, it is seen for instance that

$$
\frac{4 \mathcal{M}_{0}}{\xi} \varepsilon^{2}=\frac{4 \mathcal{M}_{0}}{\xi_{0}} \varepsilon=\frac{C_{L}^{0}}{S^{\star}} \quad \text { and } \quad \mathcal{D}=\frac{\mathcal{A}_{0} \mathcal{M}_{0}}{\xi_{0}^{2}}=\frac{\mathcal{A}_{0}}{\varepsilon \xi_{0}} \frac{C_{L}^{0}}{4 S G^{\star}}
$$

These relations allow to translate the main results of the following analysis, which are presented with the parameters of the wake-oscillator model, into more usual dimensionless numbers.

The smallness of the five parameters $\xi_{s}, \xi_{a}, \varepsilon, \alpha$ and $I_{u}$ is fully exploited in the following developments by means of a perturbation approach. This makes possible the establishment of analytical expressions for the stochastic response of the wake-oscillator model. 
Table 2

Considered numerical values used for the illustrations. Below first separation: derived quantities.

\begin{tabular}{lll}
\hline $\begin{array}{l}\text { Model } \\
\text { parameters }\end{array}$ & $\begin{array}{l}\text { Data Set } 1 \\
\text { Dimensionless } \\
\text { parameters }\end{array}$ & $\begin{array}{l}\text { Data Set } 2 \\
\text { Dimensionless } \\
\text { parameters }\end{array}$ \\
\hline$\varepsilon$ & $\varepsilon=0.05$ & $\varepsilon=0.2$ \\
$\xi_{s}$ & $\xi_{s}=0.015$ & $\xi_{s}=0.0075$ \\
$\xi_{a}$ & $\xi_{a}=0.015$ & $\xi_{a}=0.0075$ \\
$\mathcal{M}_{0}$ & $\mathcal{M}_{0}=0.9$ & $\mathcal{M}_{0}=0.0187$ \\
$\mathcal{A}_{0}$ & $\mathcal{A}_{0}=1$ & $\mathcal{A}_{0}=6$ \\
$\alpha$ & Variable & Variable \\
$I_{u}, \mathcal{I}_{0}=I_{u} / \varepsilon$ & Variable & Variable \\
\hline$\xi=\xi_{s}+\xi_{a}$ & $\xi=0.03$ & $\xi=0.015$ \\
$\xi_{0}=\xi / \varepsilon$ & $\xi_{0}=0.6$ & $\xi_{0}=0.075$ \\
$\mathcal{D}$ & $\mathcal{D}=2.5$ & $\mathcal{D}=20$ \\
\hline$\xi(1+\mathcal{D})$ & $\Delta \Omega= \pm 0.105$ & $\Delta \Omega= \pm 0.315$ \\
\hline
\end{tabular}

\section{Deterministic wake-oscillator model}

\subsection{Averaged governing equations}

When the turbulence is discarded $\left(\mathcal{U}(\tau)=0\right.$ or $\left.I_{u}=0\right)$, the set of equations boils down to the governing equations studied by Facchinetti et al. (2004),

$$
\begin{aligned}
\mathcal{Y}^{\prime \prime}+2 \xi \mathcal{Y}^{\prime}+\mathcal{Y} & =2 \varepsilon \mathcal{M}_{0} \Omega^{2} \mathcal{Q} \\
\mathcal{Q}^{\prime \prime}+\varepsilon \Omega\left(Q^{2}-1\right) Q^{\prime}+\Omega^{2} Q & =2 \varepsilon \mathcal{A}_{0} \mathcal{Y}^{\prime \prime}
\end{aligned}
$$

where $\xi=\xi_{s}+\xi_{a}$ represents the sum of structural and aerodynamic dampings. We only focus on the specific case where $\{\xi, \varepsilon\} \ll 1$ and this is formalized by introducing

$$
\xi=\xi_{s}+\xi_{a}=\xi_{0} \varepsilon
$$

where $\xi_{0} \sim 1$ (is of order 1 at most). We also focus on small mistuning conditions, i.e. assume that the vortex shedding frequency of the fixed cylinder is close to the natural frequency of the structure. This is formalized by writing

$$
\Omega=1+\xi \delta=1+\xi_{0} \varepsilon \delta \quad \Leftrightarrow \quad \delta=\frac{\Omega-1}{\xi}=\frac{f_{\text {shedding }}-f_{0}}{\xi f_{0}}
$$

where $\delta \sim 1$ is a detuning parameter of order 1 . The detuning is scaled with respect to $\xi$ which might appear unusual at first glance. In the forced case where the same problem is reconsidered by imposing a prescribed motion to the body, the structural damping ratio vanishes and the proper scaling is $\omega=(\Omega-1) / \varepsilon$, see Appendix A.2. The choice to scale here the mistuning with $\xi$, in the free vibration test, will appear natural in the light of the following results.

The set of governing Eqs. (20) is then solved by considering a multiple scales solution, in the form of the following ansatz

$$
\begin{aligned}
& \mathcal{Y}(\tau ; \varepsilon)=\mathcal{Y}_{0}[\tau, T(\tau) ; \varepsilon]+\varepsilon \mathcal{Y}_{1}[\tau, T(\tau) ; \varepsilon]+\cdots \\
& \mathcal{Q}(\tau ; \varepsilon)=\mathcal{Q}_{0}[\tau, T(\tau) ; \varepsilon]+\varepsilon \mathcal{Q}_{1}[\tau, T(\tau) ; \varepsilon]+\cdots
\end{aligned}
$$

where $\mathcal{Y}_{i}$ and $\mathcal{Q}_{i}(i=0,1, \ldots)$ are of order 1 , which indicates that the solution is sought as a function of a fast time $\tau$ and a slow time $T$ chosen as $T=\varepsilon \tau$. Application of standard techniques in multiple timescales (Bender and Orszag, 2013) shows that the leading order solution of the set of governing equations is

$$
\mathcal{Y}_{0}=R_{y}(T) \cos [\tau+\varphi(T)] \quad ; \quad \mathcal{Q}_{0}=R_{q}(T) \cos [\tau+\varphi(T)+\psi(T)]
$$

where the slowly varying amplitudes $R_{y}(T), R_{q}(T)$ and the relative phase $\psi(T)$ satisfy the secularity equations (see details in Appendix A.1)

$$
\begin{aligned}
R_{q}^{\prime} & =\mathcal{A}_{0} R_{y} \sin \psi-\frac{1}{8} R_{q}^{3}+\frac{1}{2} R_{q} \\
R_{y}^{\prime} & =\mathcal{M}_{0} R_{q} \sin \psi-\xi_{0} R_{y} \\
\psi^{\prime} & =\left(\mathcal{A}_{0} \frac{R_{y}}{R_{q}}+\mathcal{M}_{0} \frac{R_{q}}{R_{y}}\right) \cos \psi+\xi_{0} \delta .
\end{aligned}
$$

This way of averaging the governing equations reveals that the 4-dimensional dynamical system described by the original Eqs. (11) in the state-space actually evolves on a 3-dimensional subspace, at leading order. This is consistent 
with the usual features of multiple scale analysis in the time (Bender and Orszag, 2013) or frequency (Denoël, 2015) domains.

The multiple scale analysis that has resulted in the averaged Eqs. (25) has some advantages compared to assuming delayed harmonic responses for $\mathcal{Y}$ and $\mathcal{Q}$ as in Facchinetti et al. (2004): (i) it explicitly introduces the slow amplitude and slow phase, (ii) it also explicitly introduces the governing equation for the dynamics of the slow phase, the third equation in (25), which is a central concept in synchronization, (iii) final results take simple expressions since they correspond to secularity conditions instead of long derivations involving trigonometry and algebra. On the flip side, the mistuning needs to be small.

\subsection{The slow phase on the limit cycle}

The steady state solution is obtained when the time derivatives on the lefthand sides in (25) vanish. In particular the second equation yields $\mathcal{M}_{0} R_{q} \sin \psi=\xi_{0} R_{y}$, so that the ratio

$$
\frac{R_{y}}{R_{q}}=\frac{\mathcal{M}_{0}}{\xi_{0}} \sin \psi
$$

is known and its substitution into the last equation indicates that the phase on the limit cycle $\cot \psi_{\mathrm{LC}}$ thus satisfies

$$
\cot ^{3} \psi_{\mathrm{LC}}+\delta \cot ^{2} \psi_{\mathrm{LC}}+\left(1+\frac{\mathcal{A}_{0} \mathcal{M}_{0}}{\xi_{0}^{2}}\right) \cot \psi_{\mathrm{LC}}+\delta=0 .
$$

If the lefthand side $\psi^{\prime}$ had been kept, this would have provided the slow phase model in smooth oncoming flow conditions. It is not written here since only the limit cycle usually matters in smooth flow conditions. The phase on the limit cycle $\psi_{\mathrm{LC}} \equiv \psi_{\mathrm{LC}}(\delta, \mathcal{D})$ only depends on the mistuning $\delta$ and on the dimensionless group

$$
\mathcal{D}:=\frac{\mathcal{A}_{0} \mathcal{M}_{0}}{\xi_{0}^{2}}=\frac{\mathcal{A}_{0} \mathcal{M}_{0}}{\xi^{2}} \varepsilon^{2}=\frac{2 \mathcal{A}_{0}}{\varepsilon} \frac{1}{2 \xi} \frac{\rho U_{\infty}^{2} C_{L}^{0}}{8 k_{\mathrm{S}}}=\frac{\mathcal{A}_{0}}{\varepsilon \xi_{0}} \frac{C_{L}^{0}}{4 S G^{\star}} .
$$

This group translates the relative magnitude between the two (small) forcing terms in the coupled governing equations.

Since the mistuning parameter $\delta$ appears in all even powers of $\cot \psi_{\mathrm{LC}}$ and only there, at leading order, the phase on the limit cycle $\psi_{\mathrm{LC}}$ is symmetric with respect to $\delta$, i.e. not affected by a change of sign of $\delta$. As a consequence, the lock-in domain is symmetric with respect to the reduced velocity. This is an important feature of the Fdlb model.

General solution. If $\delta=0$, the polynomial (27) in $\cot \psi_{\mathrm{LC}}$ has only one real root (because $\mathcal{D}>0$ ) corresponding to $\cot \psi_{\mathrm{LC}}=0$, i.e. $\psi_{\mathrm{LC}}= \pm \frac{\pi}{2}$, which corresponds to a perfect lock-in condition. Indeed, as seen next, this maximizes the response of the system (it is possible to anticipate that a phase shift of $\pm \frac{\pi}{2}$ maximizes the energy pumped into the structural degree-of-freedom). Invoking Descartes' rule of sign, it is observed that the polynomial (27) has one or three positive roots if $\delta<0$ and one or three negative roots if $\delta>0$. Furthermore, using Cardano's formula, it is possible to prove that, if $\mathcal{D}<8$, there is only one real root no matter the value of $\delta$. On the contrary, if $\mathcal{D}>8$, there are three roots if $|\delta|$ lies in the interval $\left[I^{(-)} ; I^{(+)}\right]$and only one root otherwise. The bounds of this interval are given by

$$
I^{( \pm)}=\frac{\sqrt{\mathcal{D}^{2}+20 \mathcal{D}-8 \pm \sqrt{\mathcal{D}(\mathcal{D}-8)^{3}}}}{2 \sqrt{2}} .
$$

The upper bound can be approximated by $I^{(+)} \simeq(4+\mathcal{D}) \frac{\sqrt{3}}{4}$ for values of $\mathcal{D}$ in the range [8, 20], which corresponds to typical applications.

The existence of three real roots is associated with some hysteresis in the model (Païdoussis et al., 2010). Fig. 1 represents the one or three roots of the polynomial equation as a function of $\delta$ and for various values of $\mathcal{D}$. The figure indeed illustrates that there is only one root for $\mathcal{D}<8$ and therefore no hysteresis (blue lines), while there might be three real roots and hysteresis in the system for $\mathcal{D} \geq 8$ (red lines).

Exact solutions of the 3rd degree polynomial (27) can be obtained by means of Cardano's formula. The resulting expressions are rather long; they are not reported here. Instead we focus on simple approximate solutions.

Small-root asymptote (Bulk of lock-in region). Let us examine first the conditions under which it is possible to have a small root. This case is the most important since it corresponds to the lock-in phenomenon, $\left|\cot \psi_{\mathrm{LC}}\right| \ll 1$ meaning $\psi \simeq \pm \frac{\pi}{2}$. A small-root asymptote is obtained by dropping terms in $\cot ^{2} \psi_{\mathrm{LC}}$ and $\cot ^{3} \psi_{\mathrm{LC}}$ in (27). This yields

$$
\cot \psi_{\text {small }} \simeq \frac{-\delta}{1+\mathcal{D}} .
$$

This approximation ceases to be valid when either $\cot ^{3} \psi_{\mathrm{LC}}$ either $\delta \cot ^{2} \psi_{\mathrm{LC}}$ enters in the balance in (27), that is when either (i) $\cot ^{3} \psi_{\mathrm{LC}} \sim \delta$, i.e. $\left(\frac{\delta}{1+\mathcal{D}}\right)^{3} \sim \delta$ or $|\delta| \sim(1+\mathcal{D})^{3 / 2}$, either (ii) $\delta \cot ^{2} \psi_{\mathrm{LC}} \sim \delta$, i.e. $|\delta| \sim 1+\mathcal{D}$. All in all, the small-root asymptote is therefore valid when $|\delta| \ll 1+\mathcal{D}$. The small-root asymptote is represented by dashed lines in Fig. 1; this root is represented in the range of validity, $|\delta| \lesssim 1+\mathcal{D}$. 


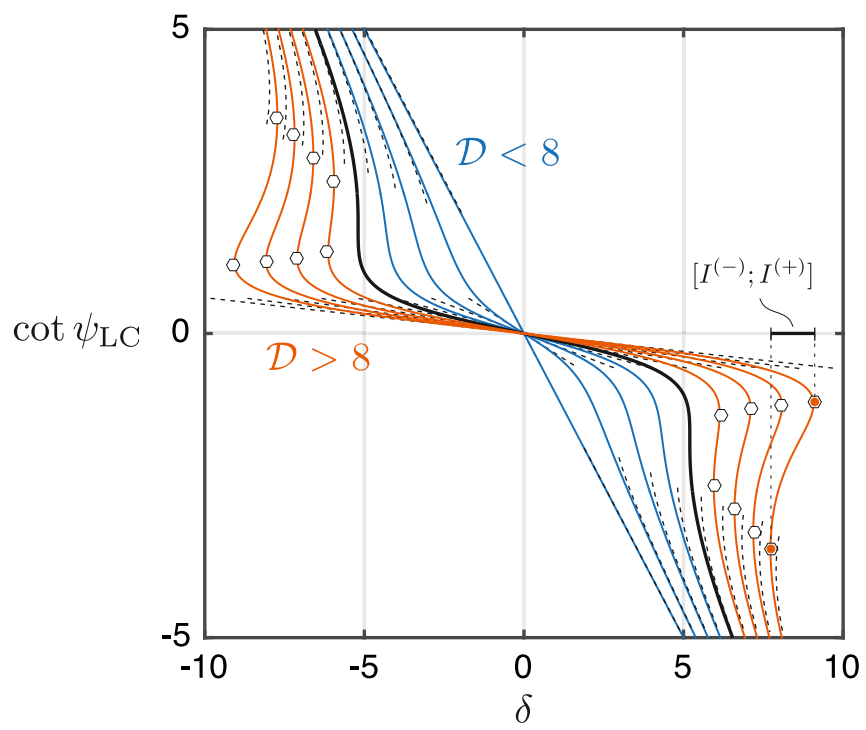

Fig. 1. Steady-state phase between wake and oscillator on the limit cycle. There are three possible solutions for $\mathcal{D} \geq 8$ (in red) and just one solution for $\mathcal{D}<8$ (in blue). Represented for $\mathcal{D}=0,2,4,6$ (in blue), $\mathcal{D}=8$ (in black), $\mathcal{D}=10,12,14,16$ (in red). Dashed line represent the small-root and large-root asymptotes. (For interpretation of the references to color in this figure legend, the reader is referred to the web version of this article.)

Large-root asymptote. For $|\delta|>I^{(+)}$, far from the center of the lock-in region, there is only one large root, and the root can be approximated by the solution of $\cot ^{3} \psi+\delta \cot ^{2} \psi=0$, i.e. $\cot \psi+\delta=0$. Reconsidering the polynomial (27) we observe that, in the limit case, the independent term balances the term $\cot \psi$ so that we are left with $\cot ^{3} \psi_{\mathrm{LC}}+\delta \cot ^{2} \psi_{\mathrm{LC}}+\mathcal{D} \cot \psi_{\mathrm{LC}}=0$ whose solution is

$$
\cot \psi_{\text {large }} \simeq \frac{-\delta \pm \sqrt{\delta^{2}-4 \mathcal{D}}}{2} .
$$

This is the asymptotic solution far from the bulk of the lock-in region. It is just given here for information; it is not used in the sequel since it corresponds to a regime that falls out of the lock-in range and is therefore less interesting. This approximate solution is also represented by dashed lines in Fig. 1.

The extent of the lock-in region. The range of validity of the small-root corresponds to the domain where the fluid and structure oscillators find a steady state solution with a phase shift close to $\pi / 2$, indicating that there is a significant energy exchange between the two oscillators, the fluid one being able to store energy and resulting therefore in large structural oscillations. This translates the so-called lock-in phenomenon observed in vortex-induced vibrations. The size of the lock-in region corresponds therefore to the region where the small-root asymptote is accurate, i.e. $|\delta| \ll 1+\mathcal{D}$. With dimensional parameters, it translates into $\Delta \Omega \ll \xi(1+\mathcal{D})$. This solution is however only valid under the condition $\mathcal{D}<8$, where $\cot \psi_{\mathrm{LC}}$ grows monotonically with $-\delta$. Indeed, in the case $\mathcal{D}>8$, the folding in the solution is such that the branch associated with the large root can connect the small-root asymptote before it becomes large. In that case, the extent of the lock-in region is precisely given by the upper bound of the interval $\left[I^{(-)} ; I^{(+)}\right]$, i.e. approximately $\delta<(4+\mathcal{D}) \frac{\sqrt{3}}{4}$.

Aggregating these two conditions (and using $\frac{\sqrt{3}}{3}$ in the first condition to make the transition from $\mathcal{D}<8$ to $\mathcal{D}>8$ continuous), the extent of the lock-in regime can be simply expressed by

$$
\Delta \Omega=\xi \Delta \delta= \begin{cases}2 \xi(1+\mathcal{D}) \frac{\sqrt{3}}{3} & \text { if } \mathcal{D} \leq 8 \\ 2 \xi(4+\mathcal{D}) \frac{\sqrt{3}}{4} & \text { if } \mathcal{D} \geq 8\end{cases}
$$

This proves, as introduced earlier, that the extent of the lock-in region scales with the total damping ratio $\xi=\xi_{s}+\xi_{a}$ and that the parameter $\mathcal{D}$, related to the relative amplitude of the coupling terms, finely quantifies this extent. Because $\mathcal{D}$ is expressed as the product of $\mathcal{A}_{0}$ and $\mathcal{M}_{0}$, it is sufficient that one of the two righthand sides in the governing equations vanishes to obtain $\mathcal{D}=0$, i.e. the largest gradient in $\cot \psi_{\mathrm{LC}}$ vs. $\delta$, see Fig. 1, and therefore the smallest lock-in domain. The actual influence of the damping ratio on the size of the lock-in domain is actually misleading in this dimensionless formulation. Substituting in (32) the definition of $\mathcal{D}$, i.e. $\xi \mathcal{D}=\mathcal{A}_{0} \varepsilon^{2} \mathcal{M}_{0} / \xi$, this is also equivalent to

$$
\Delta \Omega=\xi \Delta \delta= \begin{cases}2 \frac{\sqrt{3}}{3}\left(\xi+\frac{\mathcal{A}_{0} \varepsilon^{2} \mathcal{M}_{0}}{\xi}\right) & \text { if } \mathcal{D} \leq 8 \\ 2 \frac{\sqrt{3}}{4}\left(4 \xi+\frac{\mathcal{A}_{0} \varepsilon^{2} \mathcal{M}_{0}}{\xi}\right) & \text { if } \mathcal{D} \geq 8\end{cases}
$$


In the most usual cases where $\mathcal{D} \gg 1$ (or even $\mathcal{D}>8$ ), the second term in the brackets leads even for moderate to large damping ratios, and the extent of the lock-in regime $\Delta \Omega$ tends to decrease with increasing damping. This is consistent with experimental evidences, see e.g. Marra et al. (2015) in air and Soti et al. (2018) in water.

Also, as the total structural damping decreases, the width of the peak in the frequency response function of the structural oscillator decreases and the bandwidth over which it is able to interact with the fluid oscillator decreases. This explains why, as $\xi$ tends to zero, the size of the lock-in domain is reduced. This might have looked paradoxical; it is important to notice however that the amplitude of the resulting motion increases as $\xi \rightarrow 0$.

\subsection{The response amplitudes on the limit cycle}

The phase shift $\psi$ plays a significant role in the analysis of the limit cycle and consequently of the possible synchronization in the presence of external forcing. This naturally appears since the first equation to solve is in cot $\psi$, while the determination of $R_{q}$ and $R_{y}$ can be seen as a secondary result. Once the phase shift between the fluid and structure oscillators is known on the limit cycle, the amplitude of vibration is obtained by combining the first and second equations in (25). They give

$$
\mathcal{A}_{0} R_{y} \sin \psi=\frac{1}{8} R_{q}^{3}-\frac{1}{2} R_{q}=\frac{\mathcal{A}_{0} \mathcal{M}_{0}}{\xi_{0}} R_{q} \sin ^{2} \psi
$$

from which it is readily seen that $R_{q}$ and $R_{y}$ satisfy

$$
R_{q}=2 \sqrt{1+2 \xi_{0} \mathcal{D} \sin ^{2} \psi} \quad ; \quad R_{y}=2 \frac{\mathcal{M}_{0}}{\xi_{0}}|\sin \psi| \sqrt{1+2 \xi_{0} \mathcal{D} \sin ^{2} \psi} .
$$

In these expressions, the maximum responses $R_{q}$ and $R_{y}$ are obtained in perfect lock-in conditions (sin $\psi=1$ ). NB: we have omitted the subscript ${ }_{\mathrm{LC}}$ in $\psi, R_{q}$ and $R_{y}$ in order to lighten the notation. The minimum response of the fluid oscillator is 2, consistently with well-established theories about the van der Pol oscillator (Nayfeh and Mook, 2008). The magnitude of the structural response on the limit cycle $R_{y}$ scales with $1 / \xi_{0}$ and $\mathcal{M}_{0}$. It is also maximum when $\sin \psi \sqrt{1+2 \xi_{0} \mathcal{D} \sin { }^{2} \psi}$ is maximum, i.e. $\psi= \pm \pi / 2$. The mistuning $\delta$ affects the magnitude of the response through the value of the phase shift $\psi$ on the limit cycle. These expressions show that the maximum responses on the limit cycle, $R_{q}$ and $R_{y} / \mathcal{M}_{0}$, only depend on $\xi_{0}=\xi / \varepsilon, \mathcal{D}$ and $\delta$ (through $\psi$ ). Fig. 2 shows the amplitude of the response for three values of $\xi / \varepsilon(0.75,0.50$ and $0.25)$, for various values of $\mathcal{D} \in[4 ; 16]$, and as a function of parameter $\delta$. The black lines correspond to $\mathcal{D}=8$, the largest value of $\mathcal{D}$ for which there is only one solution. For $\mathcal{D}>8$ (red lines), the existence of several roots translates into a "mushroom" shape, as opposed to a "bell" shape for $\mathcal{D}<8$. The vertical dashed lines in the plots of $R_{y} / \mathcal{M}_{0}$ represent the extent of the lock-in domain, as defined by (32). The good agreement with the mushroom or bell shapes indicates that this proposition qualitatively does a good job for all possible values of $\xi_{0}$ and $\mathcal{D}$. We also notice that an approximate solution in the bulk on the lock-in region can be obtained by replacing $\psi$ in (34) by the small-root asymptote (30). This yields the curved dashed lines represented on the top of the response.

Together with the simple expression of the extent of the lock-in domain, this simple expression for the magnitude of the response provides a fair estimate of the amplitude of the response that could be useful for engineering applications.

The case of perfect lock-in, when $\delta=0, \psi=\frac{\pi}{2}$, is of paramount importance since it corresponds to the maximum structural response, $R_{y}=2 \frac{\mathcal{M}_{0}}{\xi_{0}} \sqrt{1+2 \xi_{0} \mathcal{D}}$. With the original parameters of the problem, we obtain

$$
\frac{y_{\max }}{D}=\varepsilon R_{y}=2 \frac{\mathcal{M}_{0}}{\xi_{0}} \varepsilon \sqrt{1+2 \xi_{0} \mathcal{D}}=\frac{C_{L}^{0}}{2 S^{\star}} \sqrt{1+\frac{\mathcal{A}_{0}}{\varepsilon} \frac{C_{L}^{0}}{2 S^{\star}}} .
$$

This result is similar to the expression obtained by Facchinetti et al. (2004) with a (single timescale) delayed harmonic ansatz. What the current multiple scale analysis has provided in addition is a complete analysis of the phase on the limit cycle and the extent of the lock-in region. Also, we emphasize that the simple expression given here is expressed as a function of the $\mathrm{SG}^{\star}$ parameter defined with $\xi=\xi_{s}+\xi_{a}$. We chose to do it so because it is the actual quantity that governs the amplitude of the response. The maximum amplitude is unbounded as $\mathrm{SG}^{\star} \rightarrow 0$ because both the structural and the aerodynamic dampings vanish. If only the structural damping vanishes, SG $\rightarrow 0$, the aerodynamic damping remains and the Griffin plot features a saturation at low structural damping. The maximum amplitude is thus obtained by replacing SG ${ }^{\star}$ by $2 \pi^{3} \mathrm{St}^{2} \xi_{a} / \mu$ in (35). The other asymptotic case, $\mathrm{SG}^{\star} \rightarrow+\infty$ shows that $y_{\max } / D$ scales as $\left(\mathrm{SG}^{\star}\right)^{-3 / 2}$, which is consistent with experimental data, see e.g. Skop and Balasubramanian (1997), where the slope $-3 / 2$ is visible in the log-log plot.

Eqs. (32) and (35) prove very useful in identifying the parameters of the Fdlb model from experimental data. Once $\xi$ is known, the measured extent of the lock-in domain yields $\mathcal{D}$ through (32); then (35) consists in an additional equation to determine $\varepsilon$ and $\mathcal{M}_{0}$ once $y_{\max } / D$ is known.

\subsection{Period of the limit cycle}

The period of the limit cycle is obtained by returning to the leading order solution given in (24), i.e. $\mathcal{Y}_{0}=$ $R_{y}(T) \cos [\tau+\varphi(T)]$ and $\mathcal{Q}_{0}=R_{q}(T) \cos [\tau+\varphi(T)+\psi(T)]$. On the limit cycle, $R_{q}, R_{y}$ and $\psi$ have converged to steady state 

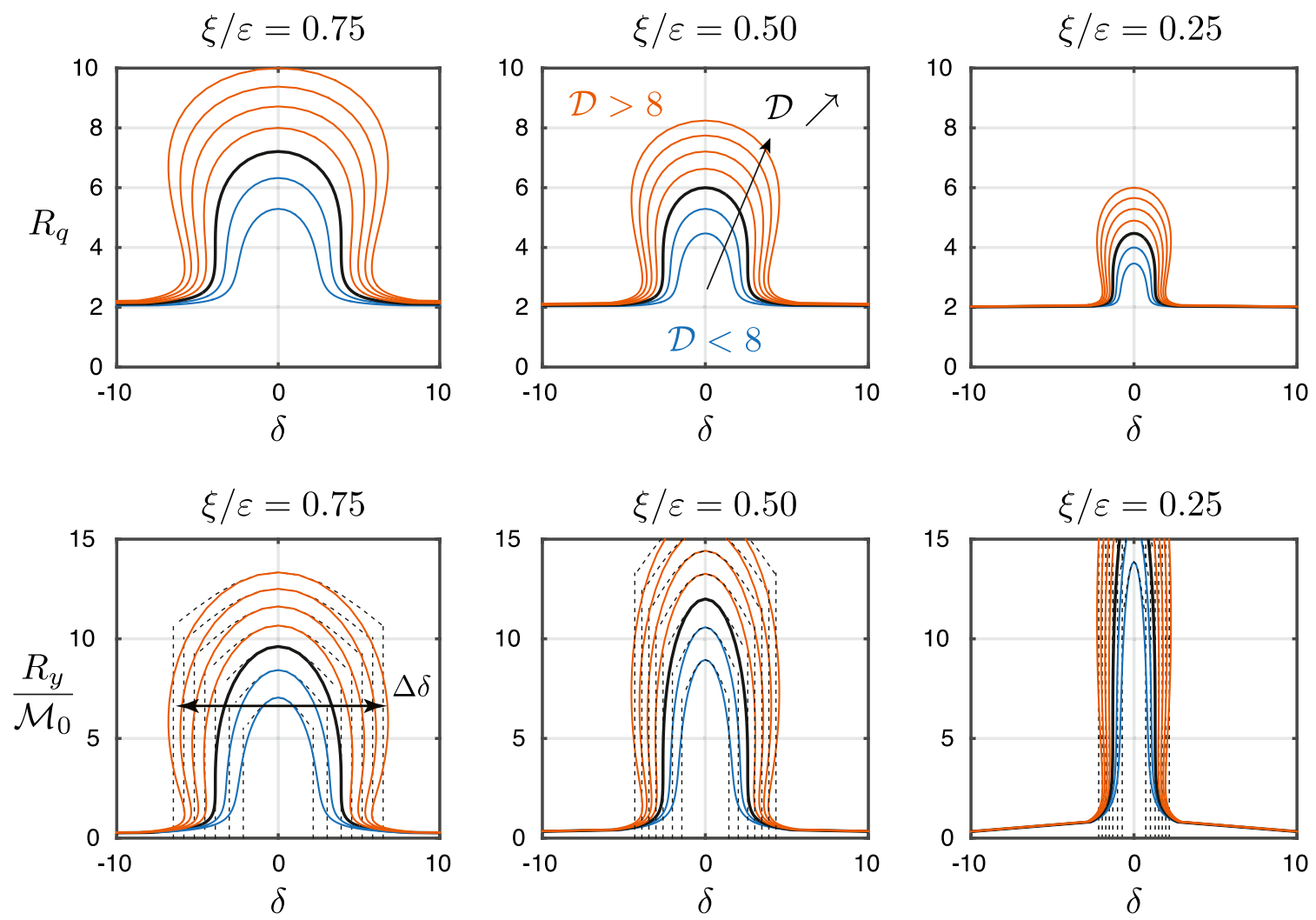

Fig. 2. Maximum responses $R_{q}$ and $R_{y} / \mathcal{M}_{0}$ on the limit cycle, represented for three values of $\xi_{0}=\xi / \varepsilon$ and for $\mathcal{D}=4,6$ (in blue), $\mathcal{D}=8$ (in black), $\mathcal{D}=10,12,14,16$ (in red). Dashed line represent the extent and height of the lock-in region, given by $(32)$ and $(34)$ where cot $\psi=-\delta /(1+\mathcal{D})$. (For interpretation of the references to color in this figure legend, the reader is referred to the web version of this article.)

values (they become constants with respect to $T$ ); on the contrary the additional phase $\varphi(T)$ might still be (slow-)time dependent. It actually satisfies

$$
\varphi^{\prime}=-\mathcal{M}_{0} \frac{R_{q}}{R_{y}} \cos \psi,
$$

see last set of equations in Appendix A.1. In fact, among the four state variables $\varphi$ is the only one that has an uncoupled slow dynamics. This is precisely an indication that the period of the limit cycle is not exactly equal to $2 \pi$. Once $R_{q}, R_{y}$ and $\psi$ have converged to the limit cycle values, satisfying (26) in particular, the previous equation provides

$$
\varphi(T)=\varphi_{0}-\left(\mathcal{M}_{0} \frac{R_{q}}{R_{y}} T \cos \psi\right)_{\mathrm{LC}}=\varphi_{0}-\xi_{0} T \cot \psi_{\mathrm{LC}}=\varphi_{0}-\varepsilon \xi_{0} \tau \cot \psi_{\mathrm{LC}}=\varphi_{0}-\xi \tau \cot \psi_{\mathrm{LC}}
$$

Substitution into $\mathcal{Y}_{0}$ and $\mathcal{Q}_{0}$ provides an argument for the cosine function different from $\tau$, which indicates that the period for a complete revolution on the limit cycle is

$$
T_{\mathrm{LC}}=\frac{2 \pi}{1-\xi \cot \psi_{\mathrm{LC}}} \simeq 2 \pi\left(1+\xi \cot \psi_{\mathrm{LC}}\right) .
$$

It is obviously the same for both $\mathcal{Y}_{0}$ and $\mathcal{Q}_{0}$ since the coupled system evolves along a unique limit cycle.

When $|\delta| \ll 1$ (perfect tuning conditions), $\psi \simeq \frac{\pi}{2}$, the period is almost equal to $2 \pi$, it is slightly shorter for $\delta>0$ ( $\cot \psi_{\mathrm{LC}}<0$ ), slightly longer for $\delta<0$ ( $\cot \psi_{\mathrm{LC}}>0$ ). Furthermore, returning to the original variable of the problem it is observed that the period elongation/shortening due to difference between the natural frequency of the structure and the vortex shedding frequency is just governed by the damping of the structural system and the phase shift $\psi_{\mathrm{LC}}$ on the limit cycle. This, again, shows how central the phase shift is.

\subsection{Illustrations}

In order to validate the averaged equations and the slow dynamics version of the problem, the original problem (8)-(9) is simulated with two sets of parameters that are specifically chosen in order to provide a lock-in response that has a bell 


\section{ARTICLE IN PRESS}
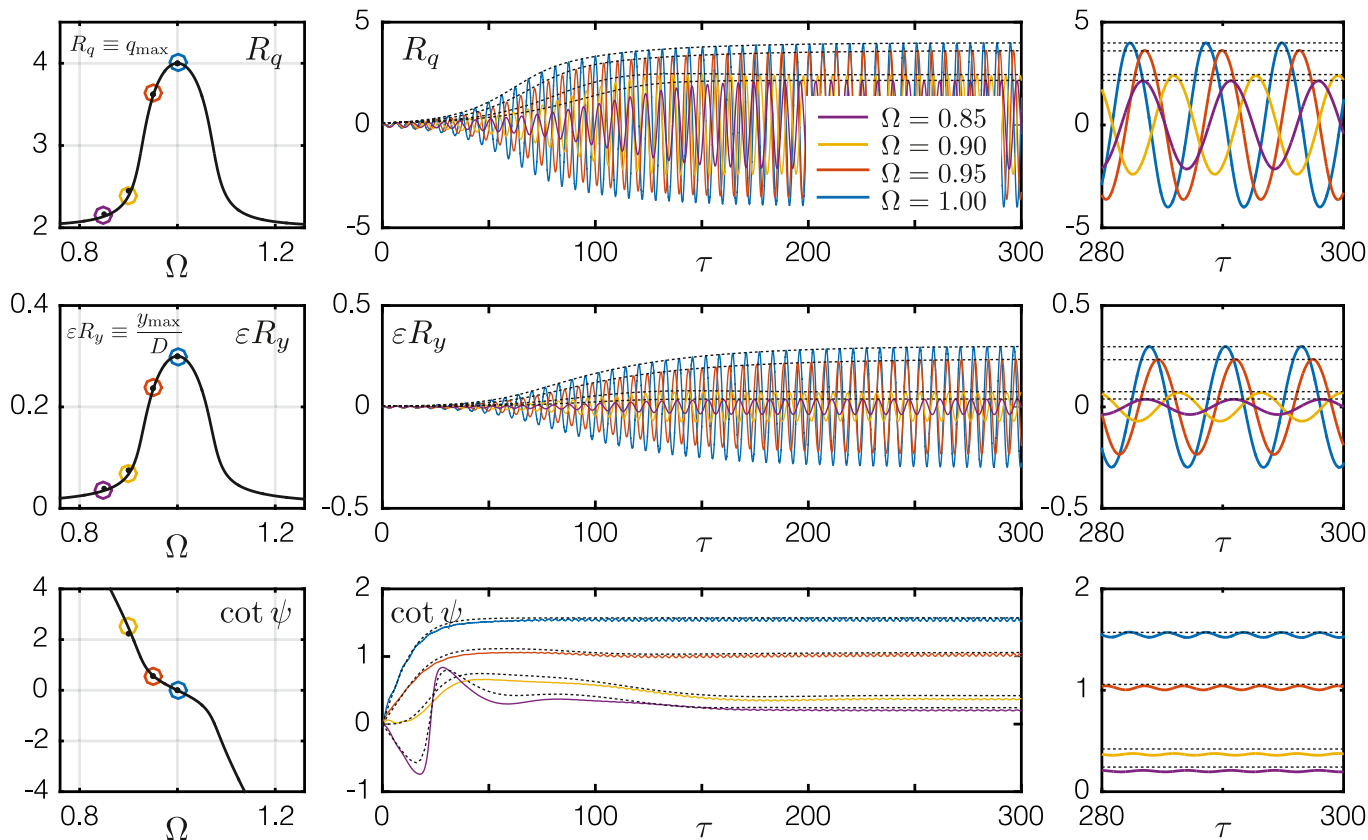

Fig. 3. Validation of the averaging of governing equations. Solution of original set of equations (colored lines) and solution of the averaged equations (black dashed lines). Numerical values correspond to Data set 1, see Table 2. Please refer to online version for colors.
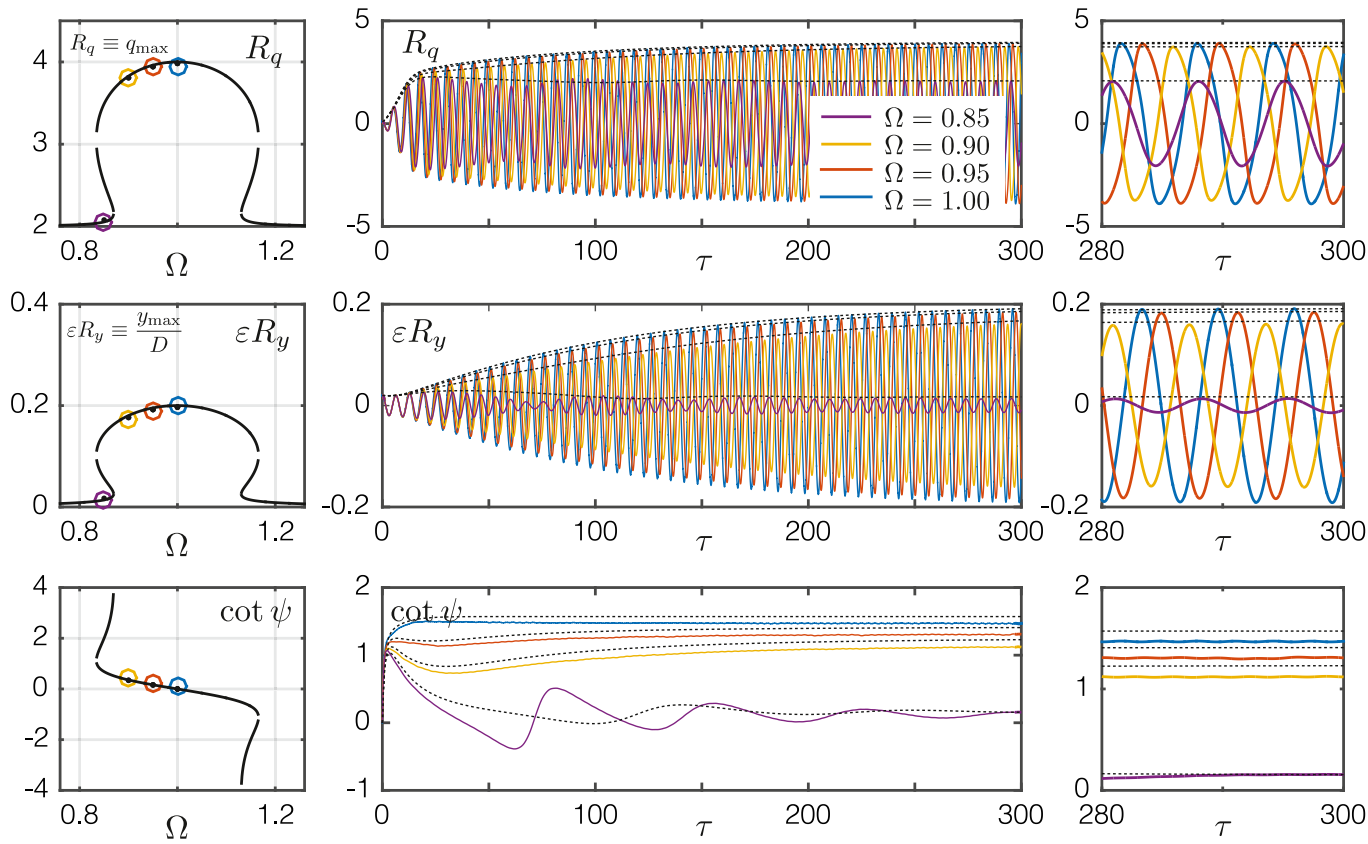

Fig. 4. Validation of the averaging of governing equations. Solution of original set of equations (colored lines) and solution of the averaged equations (black dashed lines). Numerical values correspond to Data set 2, see Table 2. Please refer to online version for colors.

shape in one case (Data Set 1) and a mushroom shape in the other (Data Set 2). The numerical values of the parameters are reported in Table 2 for the two considered cases. In the original problem, the fast dynamics is resolved and the time step $\Delta \tau$ needs to be substantially smaller that the period of the response (approx. $2 \pi$ ) in order to provide accurate results. In this illustration, they have been obtained with the default settings of the ODE45 solver that is implemented in Matlab (2012). They resulted in $\mathcal{Q}(\tau)$ and $\mathcal{Y}(\tau)$ represented by the colored lines in Figs. 3 and 4. A closeup view shows the established steady state solution in the time window $\tau \in[280,300]$. The maximum responses for each degree-of-freedom 


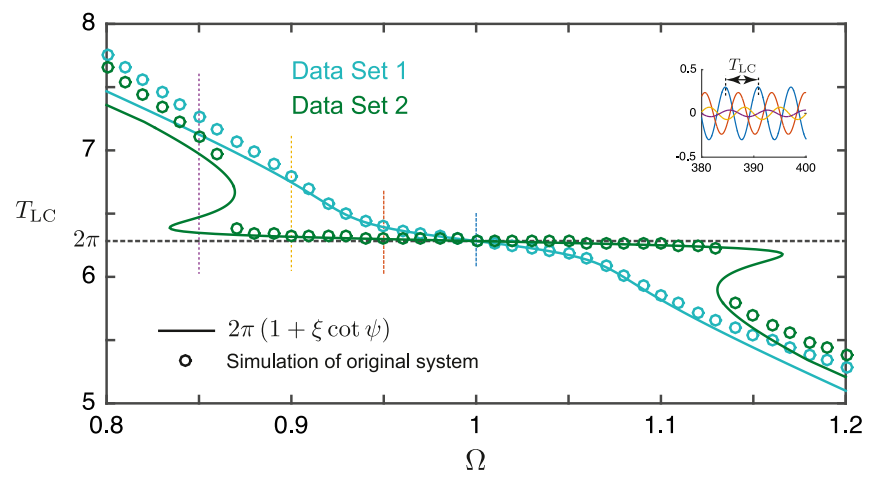

Fig. 5. Period of the limit cycle. Comparison of the period obtained from simulation of the original 4-dimensional system (time between two successive maxima) and the solution obtained after averaging (38).

are also reported as circles on the left, where they are superimposed with the analytical solution (35) of the problem (black continuous line). The time series of $\mathcal{Q}(\tau)$ and $\mathcal{Y}(\tau)$ are also Hilbert-transformed (on mirrored signals in order to limit the undesired end effects) in order to determine the instantaneous phase of the signals. The relative phase between $\mathcal{Q}(\tau)$ and $\mathcal{Y}(\tau)$ is therefore computed. It is reported with the colored lines in the bottom of Figs. 3 and 4.

The thin dashed lines represent the numerical solution of the averaged Eqs. (25). Because they just need to capture the slow dynamics of the problem, they can be computed with a much larger time step (of the order of magnitude of $\varepsilon^{-1}$ times larger), which constitutes a substantial computational saving.

In both cases the response envelopes are very well captured by the averaging procedure. The quality of the multiple timescale approach is a little worse in the second case. This is a consequence of a larger value of the "small" parameter, $\varepsilon=0.2$. In particular, it is seen that the phase is not accurately modeled for Data Set 2 and $\Omega=0.85$. In that case, the nonlinearity is higher (than for Data Set 1), and the response exhibits a strong beating which is difficulty captured by the averaging procedure. Apart from this case of lesser interest since it corresponds to small vibration amplitudes, the global response, including the transient regimes, is reasonably well estimated.

Fig. 5 shows a comparison of the period of the limit cycle obtained by simulation of the original problem (8)-(9) and with the simple analytical expression (38). In the former case, results are represented by circles and obtained from the time series of $\mathcal{Q}(\tau)$ and $\mathcal{Y}(\tau)$ by evaluating the time lapse between successive maxima in the steady state regime. The comparison is provided for many values of the mistuning $\Omega$, in the range [0.8; 1.2$]$, including the four particular values $\{0.85,0.9,0.95,1\}$ that have been used in Figs. 3 and 4 . The agreement between the results provided by the two approaches validates the statement that the period of the limit cycle is governed by $\xi$ and cot $\psi_{\mathrm{LC}}$ the cotangent of the phase on the limit cycle.

\subsection{Deterministic VIV: A synchronization phenomenon or not?}

Synchronization is a phenomenon where either (i) an oscillator with a limit cycle is externally forced by a periodic loading and phase entrainment can take place (Pikovsky et al., 2003), (ii) either several oscillators with limit cycles of similar periods of revolution might lock depending on the magnitude of the coupling terms between them (Pikovsky et al., 2003). A phenomenon is not related to synchronization as long as one is interested in the coupled system without external (time varying) forcing.

Based on this definition, it is seen that the forced response of a cylinder, as described in Appendix A.2, corresponds to frequency locking (therefore synchronization) while the free response of a cylinder in a smooth flow is not a synchronization problem. The constant wind velocity does not count as an external forcing term; it is just a (bifurcation) parameter of the problem. It becomes a synchronization problem as soon as some additional external forcing is considered.

In short, in the study of VIV, when a forced motion is imposed to the cylinder, the dynamics in the neighborhood of the limit cycle is studied and, as soon as frequency locking conditions are satisfied, the system evolves along a (slightly, as long as $\varepsilon \ll 1$ ) perturbed limit cycle. On the contrary, when the free vibration problem is considered, as in the analysis reported here, the topology of a limit cycle in a high-dimensional space is studied. Out of the lock-in region (this is not synchronization!), the limit cycle only concerns the fluid oscillator, in the Van der Pol equation, and the amplitude of the structural motion is very small. In the so-called lock-in region, the limit cycle occupies a large space in the state space and the magnitude of the structural motion on the limit cycle is much larger. This just corresponds to the analysis of the shape/topology of a limit cycle, as a function of a bifurcation parameter $(\delta)$. This is significantly different from a synchronization problem (see Appendix A.2), in the forced case, where a stability analysis is to be performed.

In the following Section, the randomized version of the problem is considered. A random external forcing in the form of a stochastic turbulent loading is added and the free vibration problem becomes therefore a synchronization problem. 


\section{Stochastic wake-oscillator model}

\subsection{The averaged model}

In the randomized version of the governing Eqs. (11), it is observed that the turbulence plays several roles. It enters (i) as a parametric excitation in the aerodynamic damping, (ii) as a forcing term in the righthand side of the first equation, (iii) in the nonlinear term of the wake equation and (iv) in the stiffness of the wake equation, which is responsible for a change of shedding frequency. It is readily seen that the first three occurrences of the turbulence are of second order, since the corresponding terms are multiplied by $\xi_{a}$ and $\varepsilon$, which are assumed to be small numbers. Consequently, for small turbulence intensity, the turbulence only affects the shedding frequency and, at first order, the governing equations read

$$
\begin{aligned}
\mathcal{Y}^{\prime \prime}+2 \xi \mathcal{Y}^{\prime}+\mathcal{Y} & =2 \varepsilon \mathcal{M}_{0} \Omega^{2} \mathcal{Q} \\
\mathcal{Q}^{\prime \prime}+\varepsilon \Omega\left(\mathcal{Q}^{2}-1\right) \mathcal{Q}^{\prime}+\Omega^{2}\left(1+2 I_{u} \mathcal{U}\right) \mathcal{Q} & =2 \varepsilon \mathcal{A}_{0} \mathcal{Y}^{\prime \prime} .
\end{aligned}
$$

Assuming again that $\varepsilon, \xi$ and $I_{u}$ are small parameters, these two equations are just seen as a small perturbation of the linear undamped oscillator so that using a technique of averaging is expected to provide an accurate solution of this problem. The same multiple timescale approach as that derived for the deterministic case shows that the leading order solution is indeed the same as (24), while the secularity conditions become (the derivation follows the same flow as in the deterministic case, detailed in Appendix A.1)

$$
\begin{aligned}
R_{q}^{\prime} & =\mathcal{A}_{0} R_{y} \sin \psi-\frac{1}{8} R_{q}^{3}+\frac{1}{2} R_{q} \\
R_{y}^{\prime} & =\mathcal{M}_{0} R_{q} \sin \psi-\xi_{0} R_{y} \\
\psi^{\prime} & =\left(\mathcal{A}_{0} \frac{R_{y}}{R_{q}}+\mathcal{M}_{0} \frac{R_{q}}{R_{y}}\right) \cos \psi+\xi_{0} \delta+\mathcal{I}_{0} \mathcal{U}
\end{aligned}
$$

where the smallness of $I_{u}$ has been made explicit by introducing $I_{u}=\varepsilon \mathcal{I}_{0}$ where $\mathcal{I}_{0} \sim 1$. This set of equations forms the averaged model. It is the same as in the deterministic case (25), apart from the external loading $\mathcal{I}_{0} \mathcal{U}$ which has appeared in the slow phase equation. It is important to notice that, at leading order, the external forcing only affects the phase dynamics and not the envelope dynamics. Since $\mathcal{U}$ is a stochastic process, the phase $\psi(T)$ and the envelopes $R_{q}(T)$ and $R_{y}(T)$ are also stochastic processes. Solving this set of governing equations requires therefore the determination of the probability density function (PDF) of these variables as well as their higher rank properties such as the power spectral density (PSD). There are several options to do so.

As a first option, a Monte Carlo Simulation of the set of governing equations is always possible. It provides an accurate result with a reasonable amount of computational power, given the low dimensionality of this problem. This approach will be used to illustrate the concepts that are developed in this Section and serve as a reference solution.

In passing, a second option would consist in writing and solving the Fokker-Planck-Kolmogorov equation associated with this problem (Risken, 1996). This equation rules the advection-diffusion of the joint PDF between $\psi(T), R_{q}(T)$ and $R_{y}(T)$. It is less general than the Monte Carlo Simulation approach, since it requires the input process $\mathcal{U}(T)$ to be Markovian. It is not a limitation when $\mathcal{U}[T(\tau)]$ is an Ornstein-Uhlenbeck process as assumed in this work; but it is for instance very difficult to generalize to more realistic turbulence models such as that described by the PSD given in (13), since many augmentation states are required to approximate such a process with a Markov model. Simulations have revealed that the joint PDF between $\psi(T), R_{q}(T)$ and $R_{y}(T)$ can take various very complicated forms, which hints that an analytical solution of the Fokker-Planck-Kolmogorov equation seems vain and that the only practical approach to solve that equation is a numerical approach. There are numerous available numerical techniques based on finite differences (Chang and Cooper, 1970), finite elements (Spencer and Bergman, 1993) or smoothed particle hydrodynamics (Canor and Denoël, 2013), to solve this equation efficiently. They are not further discussed in this paper.

Instead and as a third option, an approximate solution will be derived in the following Section. Its accuracy will be assessed by comparison with Monte Carlo Simulations of the original Eqs. (11) and of the averaged Eqs. (40).

\subsection{The slow phase model}

Under a small perturbation, such as a small (deterministic or not) forcing term, the trajectory of the system in the phase space only slightly deviates from the unperturbed limit cycle (because it is small and the cycle is stable). Thus, in terms of magnitude, perturbations of the original system lie in the near vicinity of the stable limit cycle. Conversely, the phase perturbation can be large. A small forcing can easily drive the phase point far away along the cycle. This qualitative picture suggests a description of the perturbed dynamics with the phase variable only, resolving the perturbations transverse to the limit cycle with the help of a perturbation technique. This is done by introducing the isochrones of the problem (Guckenheimer, 1975; Winfree, 2001) and focusing on the slow phase dynamics of the problem only. This approach is common in physical sciences, in particular biology (Pikovsky et al., 2003). Its formal application in the current problem would result in $\psi^{\prime}=\left(\mathcal{A}_{0} R_{y} / R_{q}+\mathcal{M}_{0} R_{q} / R_{y}\right)_{\mathrm{LC}} \cos \psi+\xi_{0} \delta+\mathcal{I}_{0} \mathcal{U}$ where $\left(\mathcal{A}_{0} \frac{R_{y}}{R_{q}}+\mathcal{M}_{0} \frac{R_{q}}{R_{y}}\right)_{\mathrm{LC}}=-\xi_{0} \delta / \cos \psi_{\mathrm{LC}}$, 

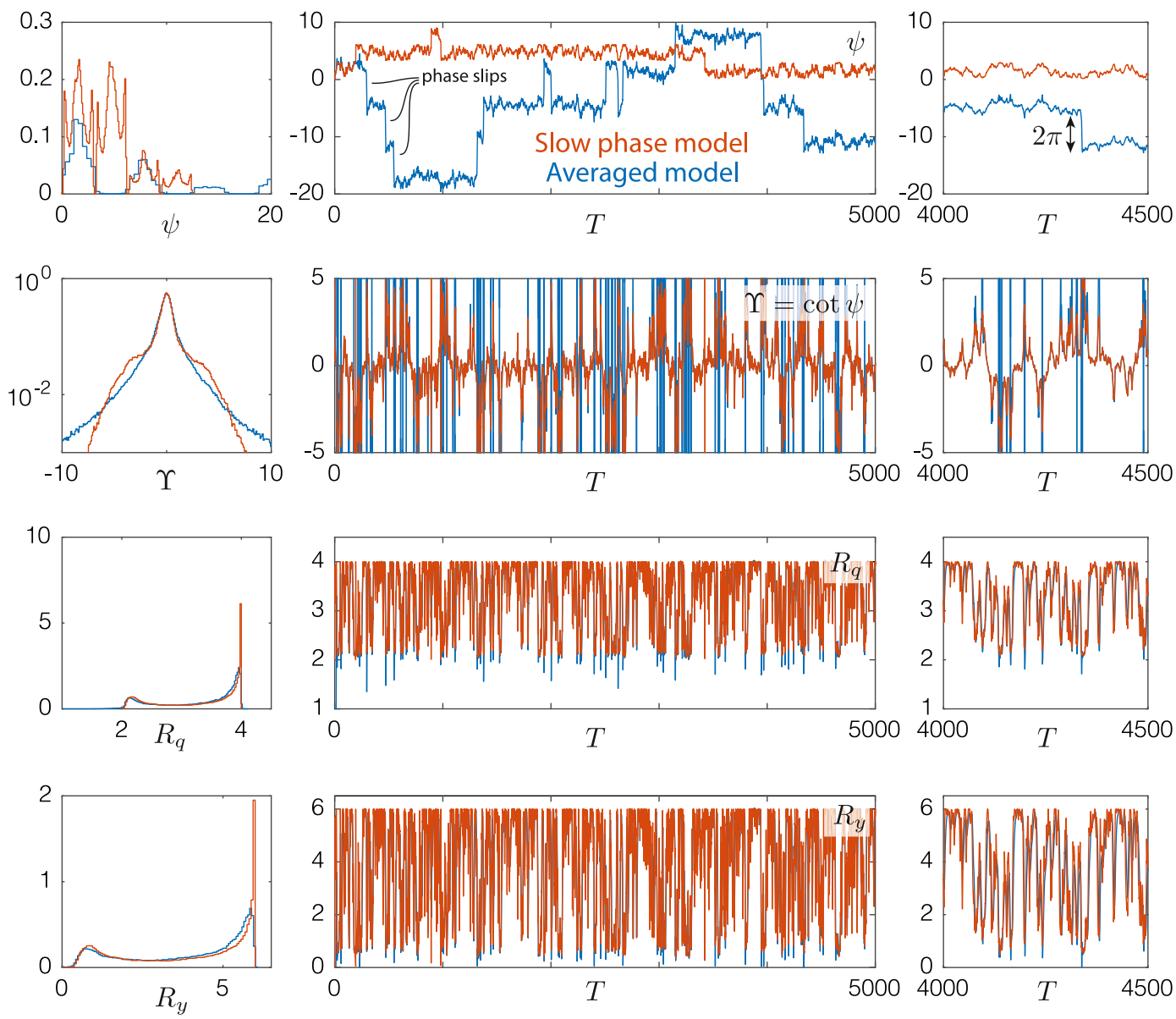

Fig. 6. Comparison of the averaged model (40) and the slow phase model (41)-(42). Left to right: normalized histograms (PDF) of $\psi, \Upsilon:=\cot \psi$, $R_{q}$ and $R_{y}$, time series, closeup view of time series. Numerical values: Data Set 1 (see Table 2), $\delta=0, \alpha=0.002$ and $I_{u}=10 \%$ (i.e. $\alpha / \varepsilon=0.04$, $\mathcal{I}_{0}=3$ ). (For interpretation of the references to color in this figure legend, the reader is referred to the web version of this article.)

see (25). The resulting equation does indeed provide a synchronization equation which unfortunately does not accept a simple explicit solution. Instead, a simple slow phase model is obtained by assuming that the relation (26) between the magnitudes of the response along the limit cycle still holds in case of a small perturbation, which corresponds to assuming that $R_{y}^{\prime}$ in the lefthand side is negligible. Its substitution into the phase equation in (40) yields

$$
\psi^{\prime}=\frac{\mathcal{A}_{0} \mathcal{M}_{0}}{\xi_{0}} \sin \psi \cos \psi+\xi_{0} \cot \psi+\xi_{0} \delta+\mathcal{I}_{0} \mathcal{U} .
$$

We have successively reduced the original 4-dimensional problem (11) to the 3-dimensional problem (40) and the arguments of small perturbation have allowed a further reduction to a 1-dimensional problem. Once the problem will be solved for the phase $\psi(T)$, the response amplitudes will be computed by

$$
R_{q}=2 \sqrt{1+2 \xi_{0} \mathcal{D} \sin ^{2} \psi} \quad ; \quad R_{y}=2 \frac{\mathcal{M}_{0}}{\xi_{0}}|\sin \psi| \sqrt{1+2 \xi_{0} \mathcal{D} \sin ^{2} \psi}
$$

where $R_{q}(T)$ and $R_{y}(T)$ are now time dependent. They are just obtained as a memoryless transformation of the phase $\psi(T)$. The slow phase model (41)-(42) not only provides a simple, although approximate, picture of the problem but is also a good candidate to explain with simple concepts and equations the influence of turbulence on a wake-oscillator model.

Fig. 6 shows Monte Carlo Simulations of (40) and (41)-(42) in order to illustrate the differences between the averaged model and the slow phase model. The numerical values considered for this example are those of Data Set 1 , and $\delta=0$. Both systems have been simulated with the same realization of the turbulence, selected as an Ornstein-Uhlenbeck process with $\alpha=0.002$ and $I_{u}=10 \%$ (i.e. $\alpha / \varepsilon=0.04$ and $\mathcal{I}_{0}=I_{u} / \varepsilon=2$ ). The total duration of the simulation is 25000 although the first 5000 units of time are shown in the time series; a closeup is also given on the window [4000;4500]. 

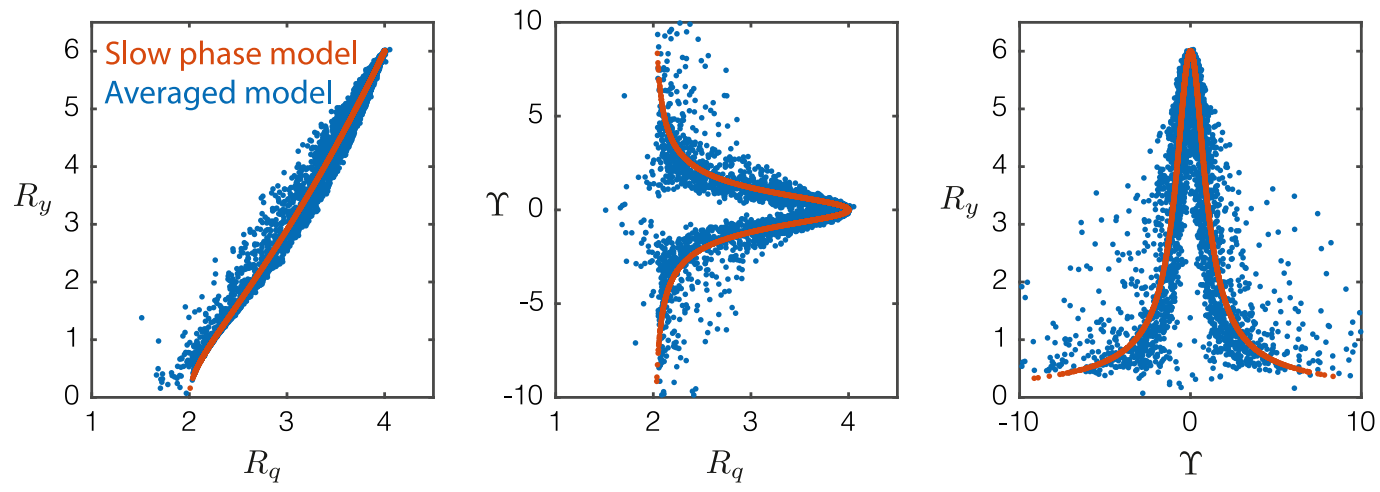

Fig. 7. Trajectories in the state space obtained with the averaged model (40) and the slow phase model (41)-(42). Numerical values: same as those in Fig. 6. Please refer to online version for colors.

The time series of $\psi(T)$ obtained with the two models look substantially different. In the averaged model (blue lines), the phase shift experiences so-called phase slips, which manifest through sudden jumps of integer multiples of $2 \pi$ in the phase shift. The accumulation of phase slips is a well-known phenomenon (Pikovsky et al., 2003) that explains the reduction of the synchronization abilities of a system when it is subject to random forced excitation. For small external forcing, the probability of occurrence of phase slips is very low; then above a certain critical magnitude of the external forcing, accumulation of phase slips occur and the rate of accumulation of phase slips further increases with the magnitude of the external forcing. The histograms of the phase slip, shown on the left of the Figure, indicate that the phase could reach values as large as 100 over the total duration of the simulation. In fact, both models are able to capture the phase slips; they just have a different critical external forcing. In the current example, we have selected the turbulence intensity $I_{u}$ so that few accumulations take place for the slow phase model (in red) while many accumulations are visible for the averaged phase model (in blue). Despite less frequent phase slips, the slow phase model actually captures quite accurately the cotangent of $\psi$, as shown in the second line of the Figure. Especially, for small values of cot $\psi$ (exactly where lock-in takes places), both models provide very similar values. This is illustrated by the superposition of the histograms of cot $\psi$ in the bulk of the domain, on the left, and by the similarity of the time series. This translates into very good approximations for $R_{q}$ and $R_{y}$ as illustrated in the bottom of Fig. 6, both in terms of time series or histograms.

The response amplitudes $R_{q}$ and $R_{y}$ are solved together with the phase in the averaged model, while they are obtained as a quasi-static post-processing of the phase in the slow phase model. In other words, the trajectories in the plane $R_{q}-R_{y}$ of the state space take place on the limit cycle in the 1-dimensional model (where the dynamics is resolved along the $\psi$ coordinate), while they can slightly scatter away from the limit cycle in the 3-dimensional model. This is illustrated in Fig. 7 showing a stroboscopic map of the trajectories $(\Delta T=6)$. This Figure illustrates that the small perturbation resulting from the turbulence is such that the system remains in the neighborhood of its natural (unperturbed) limit cycle. The limited dependence on the rate of accumulation of phase slips also indicates that isolated or repeated phase shifts do not compromise the dynamics of the system, especially as long as steady-state solutions are studied.

Eq. (41) shows that the small random perturbation acts as if the constant detuning $\xi_{0} \delta$ was replaced by a time-varying detuning $\xi_{0} \delta+\mathcal{I}_{0} \mathcal{U}$. To understand its influence on the dynamics of the slow phase and also to further illustrate the concept of phase slip, we first notice that this equation can be written

$$
\psi^{\prime}=-\frac{\mathrm{d}}{\mathrm{d} \psi} V(\psi)+\mathcal{I}_{0} \mathcal{U}
$$

where the potential $V(\psi)$ is defined as $V(\psi)=\frac{\mathcal{A}_{0} \mathcal{M}_{0}}{4 \xi_{0}} \cos 2 \psi-\xi_{0} \log |\sin \psi|-\xi_{0} \delta \psi$. This equation corresponds to the (over-)damped dynamics of a massless particle in a nonlinear potential well and subjected to a random external forcing. In the absence of external forcing $\left(\mathcal{I}_{0}=0\right)$, the system finds a stable equilibrium after the transient has vanished, in a configuration where $-V(\psi)$ is minimum. Fig. 8 shows $-V(\psi)$ for different values of the mistuning $\xi_{0} \delta$ while keeping for other parameters the same values as those considered in the Data Set 1 and Data Set 2 given in Table 2 . In the first case, a single equilibrium point exists between two successive singularities. In the second case, the existence of multiple solutions is again observed in the case $\xi_{0} \delta= \pm 0.75$, which corresponds to $\Omega=0.85$ or $\Omega=1.15$ (because $\varepsilon=0.2$ for Data Set 2), which corresponds to the purple plot in Fig. 4. In the forced configuration, the external forcing generates oscillations around the stationary point. As soon as the magnitude of the load is large enough (to be understood in a relative manner and probabilistic sense), the particle can jump to the neighboring well and drift away from its original configuration. On average, when $\delta>0$ the tendency of the drift is to the right; when $\delta<0$ the tendency of the drift is to the left.

The slow phase equation (41) can be written as a function of $\cot \psi$

$$
\psi^{\prime}=\left(\frac{\mathcal{A}_{0} \mathcal{M}_{0}}{\xi_{0}} \frac{\cot \psi}{1+\cot ^{2} \psi}+\xi_{0} \cot \psi\right)+\xi_{0} \delta+\mathcal{I}_{0} \mathcal{U}
$$



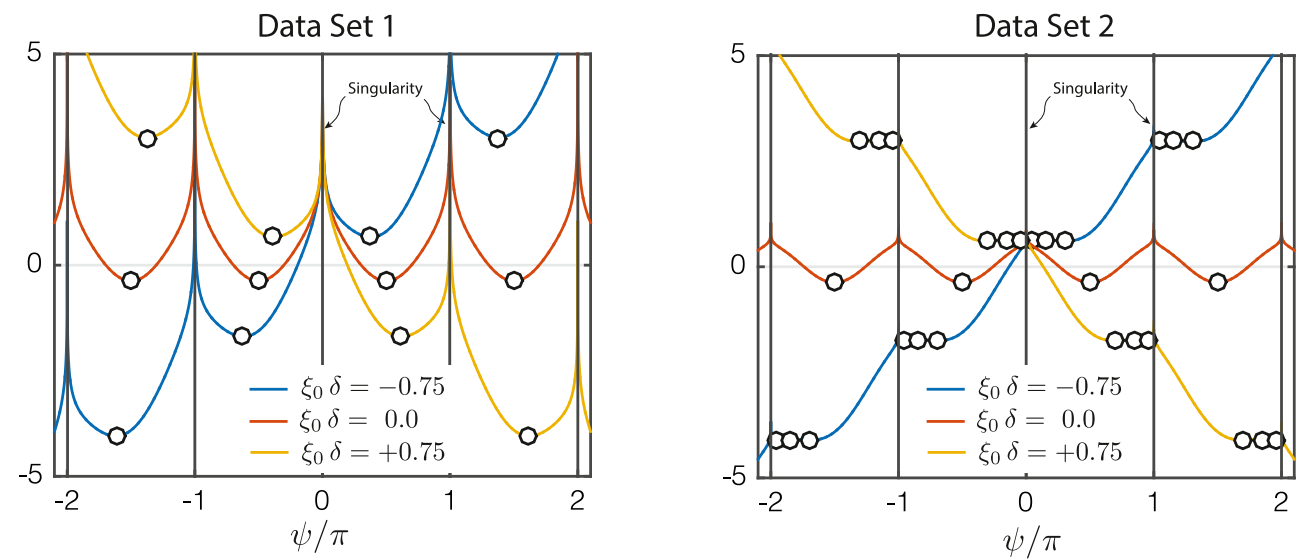

Fig. 8. Illustration of the potential $-V(\psi)$. Circles indicate local minima, i.e. stable equilibrium configurations in the absence of external forcing. Numerical values correspond to the two data sets given in Table 2. Data 1: one equilibrium point per [k $\pi,(k+1) \pi]$ interval (bell-shape lock-in response); Data 2: three equilibrium points per $[k \pi,(k+1) \pi]$ interval (mushroom-shape lock-in response). Please refer to online version for colors.

which can be further simplified by using the change of variable $\Upsilon=\cot \psi$,

$$
\frac{-\Upsilon^{\prime}}{1+\Upsilon^{2}}=\left(\frac{\mathcal{A}_{0} \mathcal{M}_{0}}{\xi_{0}} \frac{\Upsilon}{1+\Upsilon^{2}}+\xi_{0} \Upsilon\right)+\xi_{0} \delta+\mathcal{I}_{0} \mathcal{U}
$$

After rearrangement, the slow phase dynamics takes the form of a stochastic differential equation with polynomial nonlinearity and parametric excitation,

$$
\Upsilon^{\prime}+\xi_{0} \delta+\xi_{0}(1+\mathcal{D}) \Upsilon+\xi_{0} \delta \Upsilon^{2}+\xi_{0} \Upsilon^{3}=-\mathcal{I}_{0} \mathcal{U}\left(1+\Upsilon^{2}\right)
$$

Despite the apparent simplicity of this stochastic differential equation, it appears that it has no simple explicit solution. In particular, $\Upsilon$ seems to be rather Gaussian for small $\mathcal{I}_{0}$ but gets heavily lemnikurtic as soon as $\mathcal{I}_{0}$ takes some moderate values. Any approach based on stochastic linearization or closure methods seems therefore inappropriate. A naive linearization method is applied in the following Section with the objective to derive simple explicit solutions. They will be compared to accurate numerical solutions obtained with the methods suggested above for the high dimensional problem.

\subsection{Steady-state statistics of the phase shift}

An approximate solution can be obtained by expressing the cotangent of the phase $\Upsilon$ as a deviation from the cotangent of the phase on the limit cycle $\Upsilon_{\mathrm{LC}}=\cot \psi_{\mathrm{LC}}$, defined by

$$
\Upsilon_{\mathrm{LC}}^{3}+\delta \Upsilon_{\mathrm{LC}}^{2}+(1+\mathcal{D}) \Upsilon_{\mathrm{LC}}+\delta=0
$$

see (27). Indeed, with the substitution of $\Upsilon=\Upsilon_{\mathrm{LC}}+\Delta \Upsilon$ where $\Delta \Upsilon$ is a deviation (not necessarily small), the slow phase equation (46) becomes

$$
\Delta \Upsilon^{\prime}+\xi_{0}\left(1+\mathcal{D}+2 \delta \Upsilon_{\mathrm{LC}}+3 \Upsilon_{\mathrm{LC}}^{2}\right) \Delta \Upsilon+\xi_{0}\left(\delta+3 \Upsilon_{\mathrm{LC}}\right) \Delta \Upsilon^{2}+\xi_{0} \Delta \Upsilon^{3}=-\mathcal{I}_{0} \mathcal{U}\left(1+\left(\Upsilon_{\mathrm{LC}}+\Delta \Upsilon\right)^{2}\right)
$$

The advantage of this formulation is that there is no independent term in the lefthand side. As a consequence, when $\mathcal{I}_{0} \rightarrow 0$, we have $\Delta \Upsilon \rightarrow 0$, which translates into

$$
\lim _{\mathcal{I}_{0} \rightarrow 0}\left\{\mu_{\Delta \Upsilon}, \sigma_{\Delta \Upsilon}\right\}=\{0,0\}
$$

and the same for higher statistical moments. This governing equation for $\Delta \Upsilon$ remains nonlinear and its solution is not straightforward. On the lefthand side, all terms but the first one are multiplied by $\xi_{0}$; dividing through by $\xi_{0}$ and incorporating $\xi_{0}$ in a new characteristic time shows that (i) the characteristic time for the slow dynamics is in fact $\xi \tau$ (and not $\varepsilon \tau$ as used so far), (ii) the damping ratio enters in the problem on the righthand side only, in the coefficient $\mathcal{I}_{0} / \xi_{0}=I_{u} / \xi$. So the lower the damping ratio, the larger the righthand side, the larger the deviation from the unperturbed limit cycle and the further away from perfect lock-in conditions (the more phase slips occur).

The small turbulence solution. The solution of (48) is tackled in three steps. First, let us examine the case where the turbulence intensity is so small that the deviation of the phase shift from its mean value is very small, $\Delta \Upsilon \ll 1$. In that case, the governing equation collapses to

$$
\Delta \Upsilon^{\prime}+\xi_{0} \mathcal{B} \Delta \Upsilon=-\frac{\mathcal{I}_{0}}{\sin ^{2} \psi_{\mathrm{LC}}} \mathcal{U}
$$



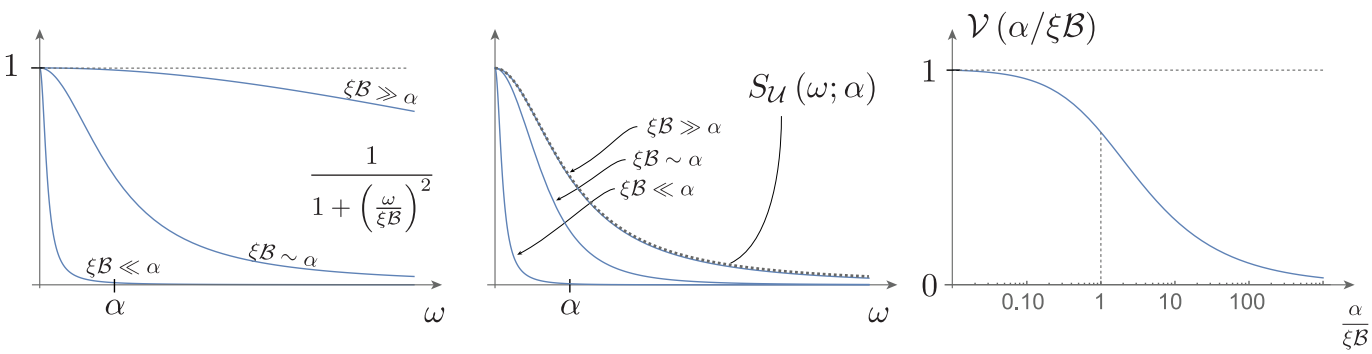

Fig. 9. Conceptual sketch illustrating the construction of $0 \leq \mathcal{V}(\alpha / \xi \mathcal{B}) \leq 1$. A lowpass filter (left) is applied to $S_{\mathcal{U}}(\omega ; \alpha)$, which results in a reduction of the high frequency content; as a consequence, $\mathcal{V}(\alpha / \xi \mathcal{B})$ is a decreasing function of $\alpha / \xi \mathcal{B}$; $\mathcal{V} \simeq 1$ for $\alpha / \xi \mathcal{B} \ll 1$ and $\mathcal{V} \ll 1$ for $\alpha / \xi \mathcal{B} \gg 1$. Please refer to online version for colors.

where $\mathcal{B}=1+\mathcal{D}+2 \delta \Upsilon_{\mathrm{LC}}+3 \Upsilon_{\mathrm{LC}}^{2}$. This linear stochastic equation possesses a simple steady state solution. Its average value is $\mu_{\Delta r}=0$ and its power spectral density (PSD) is given by

$$
S_{\Delta \Upsilon}\left(\omega_{T}\right)=\frac{\mathcal{I}_{0}^{2}}{\sin ^{4} \psi_{\mathrm{LC}}} \frac{S_{\mathcal{U}(T)}\left(\omega_{T}\right)}{\omega_{T}^{2}+\xi_{0}^{2} \mathcal{B}^{2}}
$$

where $\omega_{T}=\tilde{\omega} / \varepsilon$ is the circular frequency associated with the slow time $T$, while $\tilde{\omega}$ is the circular frequency associated with the fast (dimensionless) time $\tau$, as introduced in (14). Following similar arguments as in Section $1, S_{\mathcal{U}(T)}\left(\omega_{T}\right)$ is related to $S_{\mathcal{U}}(\tilde{\omega} ; \alpha)$ where the subscript is to be understood as $\mathcal{U} \equiv \mathcal{U}(\tau)$, by

$$
S_{\mathcal{U}(T)}\left(\omega_{T}\right)=\varepsilon S_{\mathcal{U}}\left(\varepsilon \omega_{T} ; \alpha\right)=\varepsilon \omega_{0} S_{u}\left(\varepsilon \omega_{0} \omega_{T} ; \alpha \omega_{0}\right) .
$$

The standard deviation of $\Delta \Upsilon$ is therefore given by (a subscript " ${ }_{1}$ " is added to the notation to indicate that it corresponds to the first asymptotic solution),

$$
\sigma_{\Delta \Upsilon, 1}=\frac{\mathcal{I}_{0}}{\xi_{0} \mathcal{B}} \frac{\mathcal{V}(\alpha / \xi \mathcal{B})}{\sin ^{2} \psi_{\mathrm{LC}}}
$$

where $0 \leq \mathcal{V}(\alpha / \xi \mathcal{B}) \leq 1$ is defined by

$$
\mathcal{V}^{2}\left(\frac{\alpha}{\xi \mathcal{B}}\right)=\xi_{0}^{2} \mathcal{B}^{2} \int_{-\infty}^{+\infty} \frac{S_{\mathcal{U}(T)}\left(\omega_{T} ; \alpha\right)}{\omega_{T}^{2}+\xi_{0}^{2} \mathcal{B}^{2}} \mathrm{~d} \omega_{T}=\int_{-\infty}^{+\infty} \frac{S_{\mathcal{U}}(\tilde{\omega} ; \alpha)}{1+\left(\frac{\tilde{\omega}}{\xi \mathcal{B}}\right)^{2}} \mathrm{~d} \tilde{\omega} .
$$

In usual turbulence models, the PSD of the turbulence velocity behaves as a power law for large frequencies, in the inertial range; for instance power exponents are equal to $-5 / 3$ and -2 for the two PSDs given in (13). Eqs. (51)-(52) show that the slope of $S_{\Delta r}$ for large frequencies is two units smaller than the slope of the PSD of the turbulence; $S_{\Delta r}$ decreases therefore much faster than $S_{\mathcal{U}}$ at high frequencies. This has a major influence on the variance of $\Delta \Upsilon$, which is central in understanding the loss of lock-in. With the same importance, the definition of $\mathcal{V}$ involves the product of $S_{\mathcal{U}}(\tilde{\omega} ; \alpha)$ with the frequency response function of a 1st-order filter. Therefore, the order of magnitude of $\mathcal{V}^{2}\left(\alpha, \xi_{0} \mathcal{B}\right)$ is primarily affected by the matching of the characteristic frequency (timescale) of the turbulence denoted $\alpha$ in $S_{\mathcal{U}}(\tilde{\omega} ; \alpha)$ and the cutoff frequency of the filter, denoted $\xi \mathcal{B}$. Fig. 9 illustrates the lowpass filtering effect of the PSD of the turbulence in order to obtain its influence on the stochastic phase shift. No matter the shape of the PSD $S_{\mathcal{U}}(\tilde{\omega} ; \alpha), \mathcal{V}(\alpha / \xi \mathcal{B})$ is a monotonically decreasing function, going from 1 to 0 . It transitions when $\alpha \sim \xi \mathcal{B}$.

Eq. (53) is simple and captures many of the features of the model. It is very useful once it is understood that small values of $\sigma_{\Delta \Upsilon, 1}$ mean small deviations from the limit cycle and therefore small influence of the wind turbulence on the VIV response of the body. On the contrary, larger values of $\sigma_{\Delta \Upsilon, 1}$ correspond to more frequent occurrences of phase slips and loss of synchronization. With this in mind, the model shows that

- fact 1 : if $\psi_{\mathrm{LC}}$ is close to $\pi / 2$ (center of lock-in range, $\delta \simeq 0$ ), then $\Upsilon_{\mathrm{LC}}=\cot \psi_{\mathrm{LC}}$ is close to zero and $\sin ^{2} \psi_{\mathrm{LC}}$ is close to 1 . This minimizes $\sigma_{\Delta r, 1}$ since $\sin ^{2} \psi_{\mathrm{LC}}$ comes in the denominator. If $\psi_{\mathrm{LC}}$ goes away from $\pi / 2$, then $\sin ^{2} \psi_{\mathrm{LC}}$ drops and $\sigma_{\Delta \Upsilon, 1}$ increases which results in a decrease of the VIV;

- fact 2: $\mathcal{B}$ is also typically smaller than $1+\mathcal{D}$, since $\delta$ and $\Upsilon_{\mathrm{LC}}$ have opposite signs in the center of the lock-in regime, see (30). Because $\mathcal{B}$ also comes in the denominator, $\sigma_{\Delta \Upsilon, 1}$ tends to increase when $\mathcal{B}$ decreases, as a result for instance of $\delta$ increasing.

- fact 3: $\sigma_{\Delta \Upsilon, 1}$ is proportional to $\mathcal{I}_{0}$; a larger turbulence intensity will therefore increase the phase shift and ultimately possibly reduce the VIV;

- fact 4: if $\alpha \gg \xi \mathcal{B}, \mathcal{V} \simeq 0$ and $\sigma_{\Delta r, 1}$ remains small; this means that the lock-in range and VIV response that are observed without turbulence remain unaffected by turbulence. In the limit case where turbulence is modeled as a white noise $(\alpha \rightarrow+\infty)$, there is no phase slip at all and the turbulence has no influence on the VIV response;

- fact 5: if $\alpha \ll \xi \mathcal{B}, \mathcal{V} \simeq 1$ and $\sigma_{\Delta \Upsilon, 1}$ might become larger, which translates, again, as a reduction of VIV. 


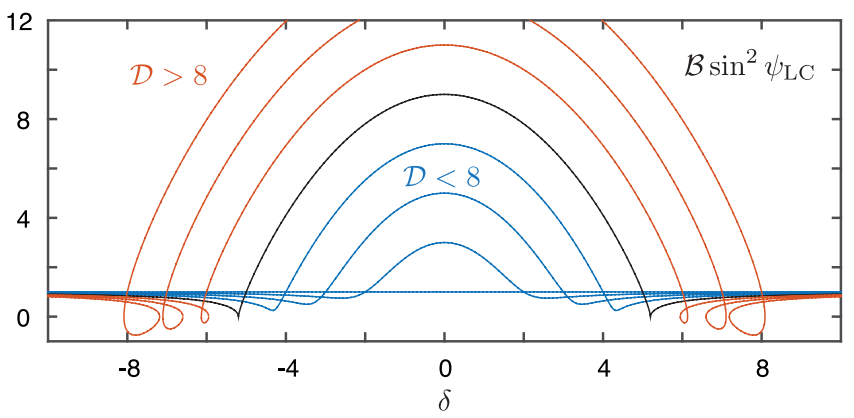

Fig. 10. Influence of $\delta$ on the small intensity solution: plot of $\mathcal{B} \sin ^{2} \psi_{\mathrm{LC}}$ as a function of $\delta$, where $\mathcal{B}(\delta)$ is defined by (47) and where $\psi_{\mathrm{LC}}$ and $\Upsilon_{\mathrm{LC}}=\cot \psi_{\mathrm{LC}}$ are given in Section 3. Please refer to online version for colors.

As a conclusion, the larger the turbulence intensity, the more turbulence is able to reduce VIV (fact 3 ) but, to do so, it is necessary that the slow timescale of the turbulence be slower than the slow characteristic time of the problem, $\alpha \ll \xi \mathcal{B}$ (fact 5). With dimensional variables, this condition reads

$$
\alpha \lesssim \xi \mathcal{B} \quad \Leftrightarrow \quad a \lesssim \xi \omega_{0}\left(1+\mathcal{D}+2 \delta \Upsilon_{\mathrm{LC}}+3 \Upsilon_{\mathrm{LC}}^{2}\right)
$$

where $a$ is the characteristic frequency of turbulence (center of gravity in the PSD of $\mathcal{U}(\tau)$ as defined in Section 1 . Furthermore, the influence of $\delta$ on both $\mathcal{B}$ (fact 2) and $\sin ^{2} \psi_{\mathrm{LC}}$ (fact 1) shows two sources of reduction of the signature of the VIV with increasing mistuning. This is also illustrated with Fig. 10 which shows $\mathcal{B} \sin ^{2} \psi_{\mathrm{LC}}$ as a function of $\delta$, in which $\mathcal{B}$ and $\sin ^{2} \psi_{\mathrm{LC}}$ themselves are expressed as functions of $\delta$.

This first solution has been obtained by neglecting the nonlinear terms in (48). This assumption is licit as long as $\sigma_{\Delta \Upsilon, 1} \ll 1$, i.e.

$$
I_{u} \ll \frac{\xi}{\mathcal{V}} \mathcal{B} \sin ^{2} \psi_{\mathrm{LC}}
$$

The large turbulence solution. Second, let us examine the case where the turbulence intensity is so large, much larger than this threshold, so that the turbulence drives the phase of the wake-oscillator model relatively far from the unperturbed limit cycle and the nonlinear governing equation needs to be solved. In that case, the two terms with the lowest powers in $\Delta \Upsilon$ can be dropped from (48) and the righthand side is also simplified to yield

$$
\Delta \Upsilon^{\prime}+\xi_{0} \Delta \Upsilon^{3}=-\mathcal{I}_{0} \mathcal{U} \Delta \Upsilon^{2} .
$$

When $\mathcal{I}_{0} \gg 1$, it is possible to find a balance between the different terms of this equation by pairing the orders of magnitude of $\xi_{0} \Delta \Upsilon^{3}$ and $\mathcal{I}_{0} \mathcal{U} \Delta \Upsilon^{2}$, which indicates that $\Delta \Upsilon \sim \mathcal{I}_{0}$ since both $\xi_{0}$ and $\mathcal{U}$ are of order 1 . Because $\mathcal{U}$ has unit variance, this shows that

$$
\sigma_{\Delta \Upsilon, 2}=\frac{\mathcal{I}_{0}}{\xi_{0}}=\frac{I_{u}}{\xi}
$$

for large turbulence intensity (a subscript " 2 " is added to the notation to indicate that it corresponds to the second asymptotic solution). This second asymptotic behavior is therefore also proportional to $\mathcal{I}_{0}$. It is however much simpler as it is now independent of $\mathcal{B}$ (but also $\mathcal{D}$ and $\delta$ ) and $\alpha$. This second solution is valid as soon as $\sigma_{\Delta r, 2} \gg 1$, i.e. $\mathcal{I}_{0} \gg \xi_{0}$ or $I_{u} \gg \xi$. Considering the typical orders of magnitude of the turbulence intensity $I_{u} \sim 10 \%-20 \%$ and of the damping ratio $\xi \sim 1 \%-5 \%$, we see that this second asymptotic case can be reached in practical applications. In fact, because it corresponds to large values of $\sigma_{\Delta r}$, it corresponds to the cases where VIV is broken by the turbulence. This simple formula suggests that turbulence hinders VIV from occurring when the turbulence intensity $I_{u}$ is one order of magnitude larger than the damping ratio $\xi$.

Solution in the transition layer. Between the two asymptotic regimes, it is difficult to find explicit solutions to the slow phase equation, since more than two (or three) terms have the same order of magnitude. In order to keep a very simple formulation, we suggest to connect the two asymptotic regimes with an equivalent linear model. It is of course less accurate than a numerical analysis of the problem, but at least it provides a simple analytical solution. If the objective was to find a very accurate solution, one would have solved the original problem.

The equivalent linear problem is obtained by linearizing the nonlinear restoring force on the lefthand side in (48) and by replacing the parametric loading on the right by an equivalent turbulence intensity. In order to follow this idea, the governing equation (48) is rewritten

$$
\Delta \Upsilon^{\prime}+f(\Delta \Upsilon)=-\mathcal{I}_{0} \mathcal{U}\left(1+\Upsilon_{\mathrm{LC}}^{2}+2 \Upsilon_{\mathrm{LC}} \Delta \Upsilon+\Delta \Upsilon^{2}\right)
$$



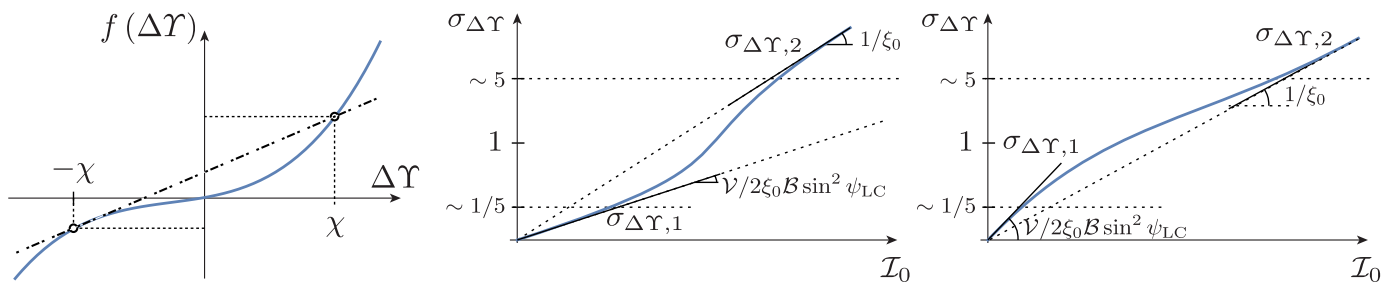

Fig. 11. Conceptual sketch illustrating the linearization of the nonlinear stochastic differential equation. Conceptual sketch illustrating the influence of the turbulence intensity on the standard deviation of the phase shift: $\sigma_{\Delta \Upsilon, 1}<\sigma_{\Delta \Upsilon, 2}$ (central plot) and $\sigma_{\Delta r, 1}>\sigma_{\Delta r, 2}$ (rightmost plot).

where $f(\Delta \Upsilon)=\xi_{0} \mathcal{B} \Delta \Upsilon+\xi_{0}\left(\delta+3 \Upsilon_{\mathrm{LC}}\right) \Delta \Upsilon^{2}+\xi_{0} \Delta \Upsilon^{3}$, and this nonlinear equation is replaced by the equivalent linear problem

$$
\Delta \Upsilon^{\prime}+\xi_{0} \beta(\Delta \Upsilon-\mu)=-\mathcal{I}_{0} \mathcal{U}\left(1+\Upsilon_{\mathrm{LC}}^{2}+\chi^{2}\right)
$$

where $\beta=\frac{1}{2 \xi_{0} \chi}[f(\chi)-f(-\chi)]$ and $\mu=\frac{-1}{2 \xi_{0} \beta}[f(\chi)+f(-\chi)]$ and have been chosen in such a way that the line $\xi_{0} \beta(\Delta \Upsilon-\mu)$ passes through the two points $(-\chi, f(-\chi))$ and $(\chi, f(\chi))$, see Fig. 11. This linear behavior $\xi_{0} \beta(\Delta \Upsilon-\mu)$ therefore replaces the nonlinear behavior $f(\Delta \Upsilon)$ in some equivalent way. This arbitrary choice yields

$$
\mu=\frac{\delta+3 \Upsilon_{\mathrm{LC}}}{1+\frac{\mathcal{B}}{\chi^{2}}} ; \beta=\mathcal{B}+\chi^{2} .
$$

We also suggest to choose $\chi=3 \sigma_{\Delta \Upsilon, 1}$ in order to represent the order of magnitude of the range covered by $\Delta \Upsilon$. The solution of the equivalent linear stochastic differential equation (60) is $\mu_{\Delta \Upsilon}=\mu$ and its power spectral density (PSD) is given by

$$
S_{\Delta \Upsilon}\left(\omega_{T}\right)=\mathcal{I}_{0}^{2}\left(1+\Upsilon_{\mathrm{LC}}^{2}+\chi^{2}\right)^{2} \frac{S_{\mathcal{U}(T)}\left(\omega_{T}\right)}{\omega_{T}^{2}+\xi_{0}^{2} \beta^{2}},
$$

which yields

$$
\sigma_{\Delta \Upsilon}=\frac{\mathcal{I}_{0}}{\xi_{0} \beta}\left(1+\Upsilon_{\mathrm{LC}}^{2}+9 \sigma_{\Delta \Upsilon, 1}^{2}\right) \mathcal{V}\left(\frac{\alpha}{\xi \beta}\right) .
$$

It is possible to check that $\lim _{\mathcal{I}_{0} \rightarrow 0} \sigma_{\Delta \Upsilon}=\sigma_{\Delta \Upsilon, 1}$ and $\lim _{\mathcal{I}_{0} \rightarrow+\infty} \sigma_{\Delta \Upsilon}=\sigma_{\Delta \Upsilon, 2}$, so that $\sigma_{\Delta \Upsilon}$ respects the two asymptotic cases. This approximation matches therefore the objectives of this derivation and provides a smooth transition between the two asymptotic cases, see Fig. 11.

Summary with dimensional quantities. In short, the cotangent of the phase shift is expressed as a deviation from the cotangent of the phase on the unperturbed limit cycle, $\Upsilon=\Upsilon_{\mathrm{LC}}+\Delta \Upsilon$. The average of $\Upsilon$ is therefore given by

$$
\mu_{\Upsilon}=\Upsilon_{\mathrm{LC}}+\frac{\delta+3 \Upsilon_{\mathrm{LC}}}{1+\frac{\xi^{2} \mathcal{B}^{3} \sin ^{4} \psi_{\mathrm{LC}}}{\left(3 I_{u} \mathcal{V}\right)^{2}}}
$$

with $\mathcal{B}=1+\mathcal{D}+2 \delta \Upsilon_{\mathrm{LC}}+3 \Upsilon_{\mathrm{LC}}^{2}$ and $\mathcal{V} \equiv \mathcal{V}(\alpha / \xi \mathcal{B})$. The standard deviation of $\Upsilon$ is $\sigma_{\Upsilon}=\sigma_{\Delta \Upsilon}$, as defined in (63).

\subsection{Steady-state statistics of the response amplitudes}

Now that the statistics of $\Upsilon(T)$ are determined, at least up second order ( $\mu_{\Upsilon}$ and $\sigma_{\Upsilon}$ ), the memoryless Eqs. (42) can be used to determine the statistics of $R_{q}(T)$ and $R_{y}(T)$. In principle $\Upsilon=\cot \psi$ is non Gaussian, see for instance illustration in Fig. 6, so the only available information (average $\mu_{\Upsilon}$ and standard deviation $\sigma_{\Upsilon}$ ) is not sufficient to fully characterize the random process $\Upsilon(T)$. However, a rough estimate of the statistics of the response amplitudes can be obtained by replacing $\Upsilon$ by a Gaussian process with the same average and standard deviation. Focusing exclusively on the structural response $R_{y}$ and replacing $\sin \psi$ in terms of $\cot \psi=\Upsilon$, the average response amplitude reads

$$
\mu_{R_{y}}=\mathbb{E}\left[R_{y}\right]=2 \frac{\mathcal{M}_{0}}{\xi_{0}} \mathbb{E}\left[\frac{\sqrt{1+\Upsilon^{2}+2 \xi_{0} \mathcal{D}}}{1+\Upsilon^{2}}\right] .
$$

Remembering that $y / D=\varepsilon \mathcal{Y}$, the average of the envelope of $y / D$ is therefore given as the product of $2 \mathcal{M}_{0} \varepsilon / \xi_{0}=C_{L}^{0} / 2 \mathrm{SG}^{\star}$, see (19), and a function of $\xi_{0} \mathcal{D}$. In the absence of turbulence, the maximum response $y_{\max } / D$ in perfect lock-in conditions is given by (35). In order to represent the influence of the turbulence with a single number, the average of the envelope 

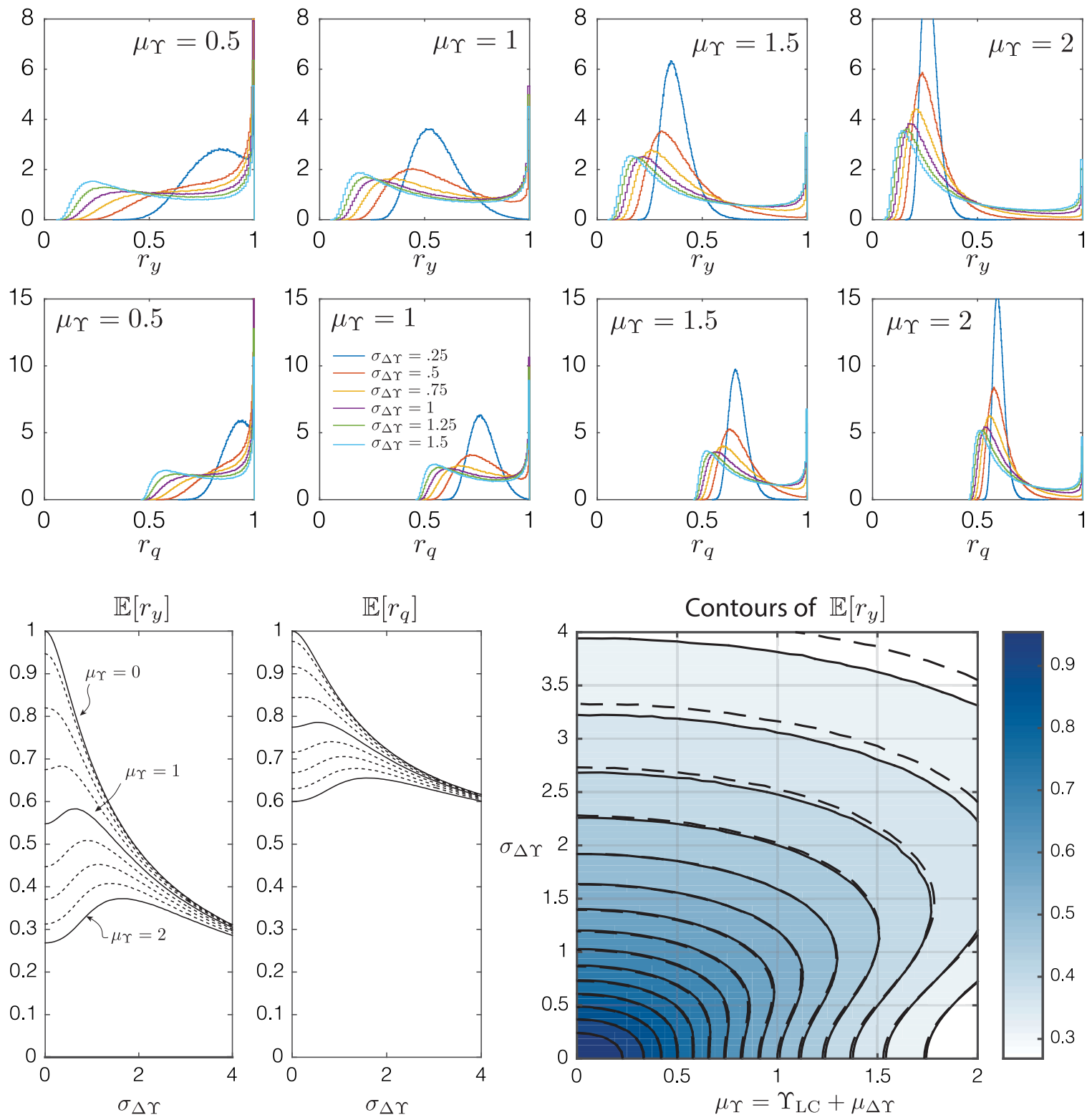

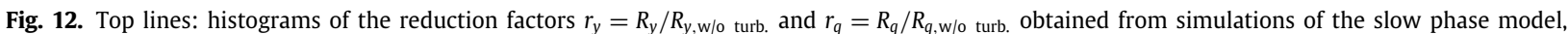
while assuming $\Delta \Upsilon=\mathcal{N}\left(\Upsilon_{\mathrm{LC}}+\mu_{\Delta \Upsilon}, \sigma_{\Upsilon}\right)$. Bottom line: average of the reduction factors $r_{y}$ and $r_{q}$ as a function of $\mu_{\Delta \Upsilon}$ and $\sigma_{\Upsilon}$. Results are obtained for $\xi_{0} \mathcal{D}=2$. Additionally, dashed lines in the contour plot correspond to $\xi_{0} \mathcal{D}=12$. Please refer to online version for colors.

of $y / D$ is divided by $y_{\max } / D$ in order to define a reduction factor translating the influence of turbulence on the structural response. It is defined by

$$
r_{y}=\frac{y / D}{y_{\max } / D}=\frac{1}{1+\Upsilon^{2}} \sqrt{\frac{1+\Upsilon^{2}+2 \xi_{0} \mathcal{D}}{1+2 \xi_{0} \mathcal{D}}} .
$$

Very similar equations hold for $r_{q}$. Fig. 12 shows the statistical distribution of the reduction factors $r_{y}$ and $r_{q}$ (first two lines) for various values of $\mu_{\Upsilon}$ and $\sigma_{\Upsilon}$. They have been obtained with a Monte Carlo simulation, assuming that $\Upsilon$ is normally distributed. These distributions confirm that when $\sigma_{\Upsilon}$ is small, the reduction factor is close to 1, i.e. the phase shift is not affected by turbulence; neither is the envelope of the response. However, as $\sigma_{\Upsilon}$ increases, the distributions of both $r_{y}$ and $r_{q}$ tend to shift to lower values, which translates the reduction of amplitudes of vortex-induced vibrations. For small $\mu_{\Upsilon}$, i.e. close to the center of lock-in, the distribution of $r_{y}$ is more spread than for large $\mu_{\Upsilon}$. See for instance the 
sharpness of the peak in the pdf of $r_{y}$ for $\mu_{\Upsilon}=2$. This indicates that the model predicts that a small turbulence intensity has more influence on the response in the center of the lock-in domain.

The statistical distributions of the reduction factors $r_{y}$ and $r_{q}$ might encapsulate too much information and it is interesting to also represent the average values $\mathbb{E}\left[r_{y}\right]$ and $\mathbb{E}\left[r_{q}\right]$ corresponding to the centroids of the distributions. Using the same numerical technique, these averages have been obtained and represented in the bottom left corner of Fig. 12, for various values of $\mu_{\Upsilon}$ and as a function of $\sigma_{\Delta \Upsilon}$. These results correspond to $\xi_{0} \mathcal{D}=2$, which seems to be a common realistic value. The same information is represented with a contour plot in the bottom right corner. Solid lines correspond to $\xi_{0} \mathcal{D}=2$; dashed lines correspond to $\xi_{0} \mathcal{D}=12$. The little difference between them indicates the slight sensitivity of the response to $\xi_{0} \mathcal{D}$, at least in the range $\xi_{0} \mathcal{D} \in[2 ; 12]$. Again, the more rapid decrease of $\mathbb{E}\left[r_{y}\right]$ with $\sigma_{\Delta r}$ for smaller values of $\mu_{\Upsilon}$ indicates that the center of the lock-in region is more affected by turbulence (in the sense of a reduction of VIV amplitude) than the outer zone of the lock-in domain. The slight sensitivity to $\xi_{0} \mathcal{D}$ indicates that the main influence of $\xi_{0}$ and $\mathcal{D}$ on the VIV responses mainly takes place through the statistics of $\Delta \Upsilon$, and not the memoryless transformation having led to (66).

The series assembly of the computation of the statistics of $\Upsilon$ by means of (64) and (63) and the determination of the average of the response envelope by means of a numerical simulation of (65) or of the graphs represented in Fig. 12 closes the loop and provide a simple efficient solution to the analysis of the Fdlb model in stochastic conditions.

\section{Numerical validation, discussion and illustrations}

In this Section, some illustrations are given to support the derivation of the slow phase model. In order to obtain this model, the original model has been averaged. The averaging procedure is not related to the external stochastic loading; it has been illustrated in Section 3. Also, the comparison of the averaged model and the slow phase model has been illustrated in Fig. 6. This Section therefore focuses on two important aspects of the model: the evaluation of the proposed approached formulas for $\sigma_{\Delta r}$ and the final comparison of the response amplitudes for all models. These two aspects are treated sequentially in this Section.

For the purpose of the illustrations, Data Set 1 is chosen and the turbulence is modeled as an Ornstein-Uhlenbeck process, i.e. the PSD of the dimensionless turbulence $\mathcal{U}(\tau)$ is given by the second equation in (15).

\subsection{Evaluation of the proposed analytical formula for the statistics of $\Delta \Upsilon$}

The derivation in Section 4.3 makes it clear that the deviation $\Delta \Upsilon$ from the limit cycle value $\Upsilon_{\text {LC }}$ plays a significant role in the attenuation of VIV. In order to evaluate the quality of the proposed analytical formulation for the standard deviation $\sigma_{\Delta r}$, the proposed formulation is compared to the reference values obtained by Monte Carlo simulations of the slow phase model. These reference values are represented by dots (Slow phase model) in Fig. 13. They have been obtained by generating samples of the turbulence that are compatible with the PSD, then solving (46) for $T \in[0,25000]$ with a slow time step $\Delta T=0.01$. Then the standard deviation of $\Delta \Upsilon$ is computed from the time series of $\Delta \Upsilon(T)$. It is reported in Fig. 13 for four different values of the turbulence intensity $I_{u} \in\{5 \%, 10 \%, 15 \%, 20 \%\}$, for three different values of $\alpha \in\{0.002,0.128,0.512\}$ and three different mistuning $\delta \in\{0, \pm 1, \pm 2\}$. Colors are use to represent different values of $\alpha$ while the three columns in the Figure correspond to the three values of $\delta$. In order to further illustrate the drift of the distribution of $\Delta \Upsilon$ from Gaussian to heavily lemnikurtic, the distributions of $\Delta \Upsilon(T)$ are also given in the same Figure. The use of a semi-log plot eases to identify the deviation from a Gaussian distribution (which would be parabolic in a semi-log plot). The typical shape of these distributions, i.e. a Gaussian distribution plus some rare excursions in the range of large phase shifts, is in agreement with the histogram of the phase that has be observed in experiments, see e.g. Zeinoddini et al. (2018). These results serve as a reference to assess the quality of the low intensity, large intensity and transition layer solutions that have been developed in Section 4.3.

For the chosen turbulence model, $\mathcal{V}=\left(1+\frac{\alpha}{\xi \mathcal{B}}\right)^{-1 / 2}$, the small and large turbulence intensity solutions become

$$
\sigma_{\Delta \Upsilon, 1}=\frac{I_{u} \csc ^{2} \psi_{\mathrm{LC}}}{\sqrt{\xi \mathcal{B}(\alpha+\xi \mathcal{B})}} \quad ; \quad \sigma_{\Delta \Upsilon, 2}=\frac{I_{u}}{\xi}
$$

where $\mathcal{B}$ is defined in (50). These two solutions are represented with dashed lines in Fig. 13. It is indeed observed that they correspond to the two asymptotic behaviors of the transition solution for each value of $\alpha$. In perfect lock-in conditions $(\delta=0), \mathcal{B}=1+\mathcal{D}=3.5$, so that $\xi \mathcal{B}=0.105$ for the considered illustration. For $\alpha \ll \xi \mathcal{B}, \sigma_{\Delta \Upsilon, 1}$ is insensitive to $\alpha$ and the response is roughly the same as that presented for $\alpha=0.002$. As soon as $\alpha \sim \xi \mathcal{B}, \alpha$ is not negligible in $\sigma_{\Delta r, 1}$ and any increase of $\alpha$ beyond $\xi \mathcal{B}$ translates in a reduction of $\sigma_{\Delta \Upsilon, 1}$, therefore in a smaller influence of the turbulence on the VIV response.

The transition from $\sigma_{\Delta \Upsilon, 1}$ to $\sigma_{\Delta \Upsilon, 2}$ which is expressed by (63) is represented by the solid lines in Fig. 13. It is observed that the transition from the small turbulence solution to the large turbulence solution is well captured by (63). For $\delta=0$ (on the left), the agreement between the proposed formulation (solid line) and the reference solution (dots) obtained with a Monte Carlo solution of (46) is very good. The accuracy of the proposed solution decreases as $\delta$ increases, i.e. further away from the center of the lock-in range, although the global trend of the increase of $\sigma_{\Delta \Upsilon}$ with $I_{u}$ is well captured. 

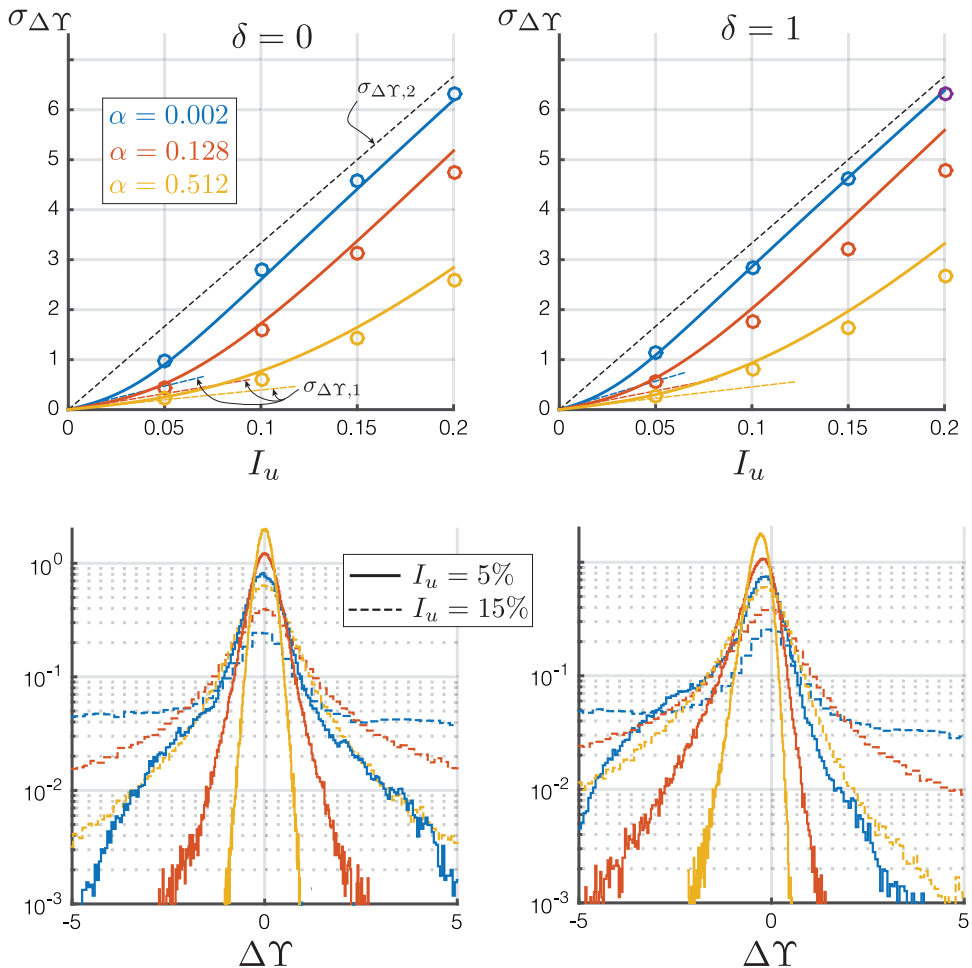
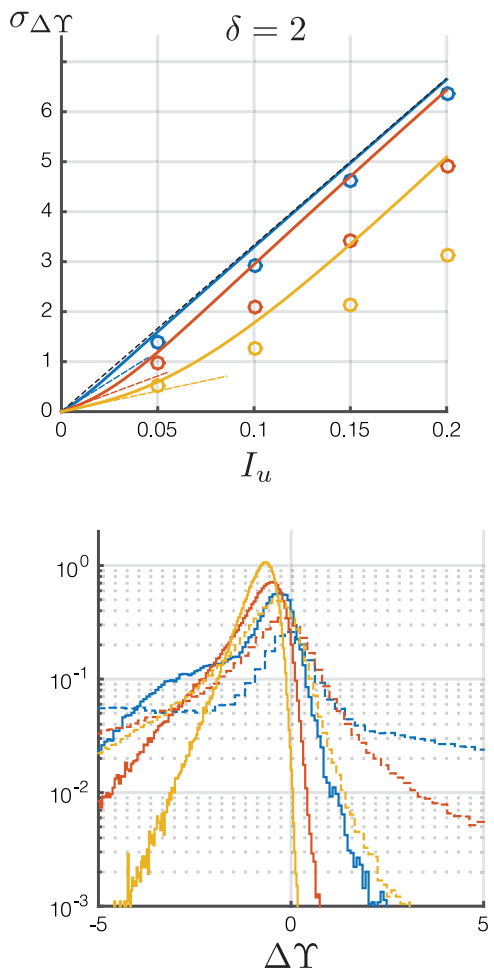

Fig. 13. Top line: standard deviation of $\Delta \Upsilon$ obtained with the slow phase model (Monte Carlo simulation of (46), dots) or with the proposed formulation (solid lines). Histograms of $\Delta \Upsilon$ illustrate the large deviations from Gaussian distributions as soon as $\alpha \ll 1$ or $\delta \gtrsim 1$. Numerical values correspond to Data Set 1.

This drop of accuracy is relatively less important as it anyway corresponds to the limits of the lock-in range, where the vibration amplitudes are smaller, even on the unperturbed limit cycle.

Globally, the distribution of $\Delta \Upsilon$ is far from being Gaussian. The complexity of the distribution of $\Delta \Upsilon$ translates the richness of the slow phase model whose solution is in fact only Gaussian when $|\delta| \ll 1$ and $I_{u} \ll 1$. It is therefore more important that the proposed model is able to capture the main trends of the response, rather than to provide an accurate result.

\subsection{Response amplitudes computed with the different models}

At last but not least, Figs. 14 and 15 show a comparison of the response amplitude computed with the three models studied in this paper as well as the proposed analytical formulation. These results have been generated with the same procedure as for Fig. 13: a sample of the turbulence that is spectrum compatible has been generated, then the governing equations are marched in time in order to obtain the time series of the responses; finally histograms of the envelope are determined with a standard descriptive statistics treatment (histograms are normalized in such a way that they do represent a density, i.e. the integral of the histograms over $\mathbb{R}$ is equal to 1 ; this makes them independent from the arbitrary bin size selection). More precisely, the original model (11) is first simulated over the time window $T \in[0,25000]$ with a time step $\Delta \tau=0.02$; in this formulation the fast dynamics are resolved; a short time step is required and this is the most time consuming model (approximately 10 to 100 times more demanding than other models). The envelope of the response for $\mathcal{Y}(T(\tau))$ is then obtained by $R_{y}=\left(\mathcal{Y}+\mathcal{Y}^{\prime}\right)^{1 / 2}$ and its histogram is established. It is compared to the response envelope $R_{y}$ obtained by Monte Carlo simulations of the averaged model (25) and of the slow phase model (41)-(42). Finally, the solution based on the analytical solution (63) is also represented. Similarly to the developments of Section 4.4, for a given set of parameters, the average and standard deviation of the phase shift are determined with (64) and (63). Samples of $\Upsilon$ are then generated, assuming Gaussianity and matching these first two moments, so that $R_{y}=2\left(\mathcal{M}_{0} / \xi_{0}\right) \sqrt{1+\Upsilon^{2}+2 \xi_{0} \mathcal{D}} /\left(1+\Upsilon^{2}\right)$ can be computed and its histogram/PDF established.

The case corresponding to $\alpha=0.002$ (first line of Figs. 14 and 15) is the most accurate. The lock-in configuration when $I_{u}=5 \%$, where the structural response is most of the time around $y_{\max } / D=0.3$, gently drifts toward a configuration where the envelope of the structural response $y_{\max } / D$ is mostly below 0.03 , when $I_{u}=10 \%$ and then $20 \%$ (from left to right in the first lines of Figs. 14 and 15). In that case, all three models as well as the proposed formulation show a very good agreement in predicting the distribution of the response amplitude. This is explained by the smallness of $R_{y}^{\prime}(T)$ which 


\section{ARTICLE IN PRESS}
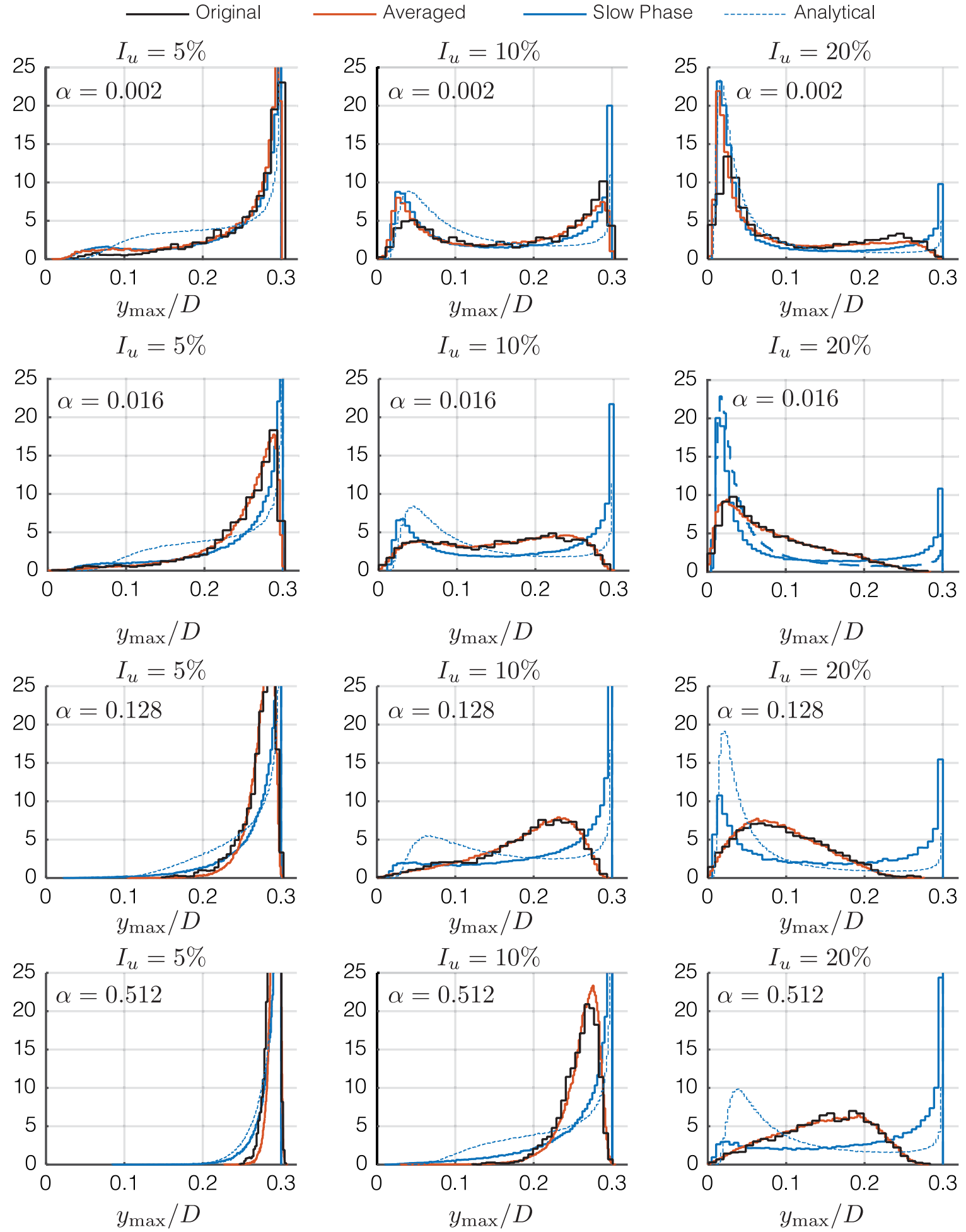

Fig. 14. Comparison of the probability density function of the envelope of the structural response $R_{y}$, obtained with the 4 models described in this paper: the original model (11), the averaged model (25), the slow phase model (41)-(42) and the analytical formulation corresponding to the small intensity asymptotic solution of the slow phase model. Numerical values : Data Set $1, \delta=0(\Omega=1), I_{u}$ variable, $\alpha$ variable. (For interpretation of the references to color in this figure legend, the reader is referred to the web version of this article.) 

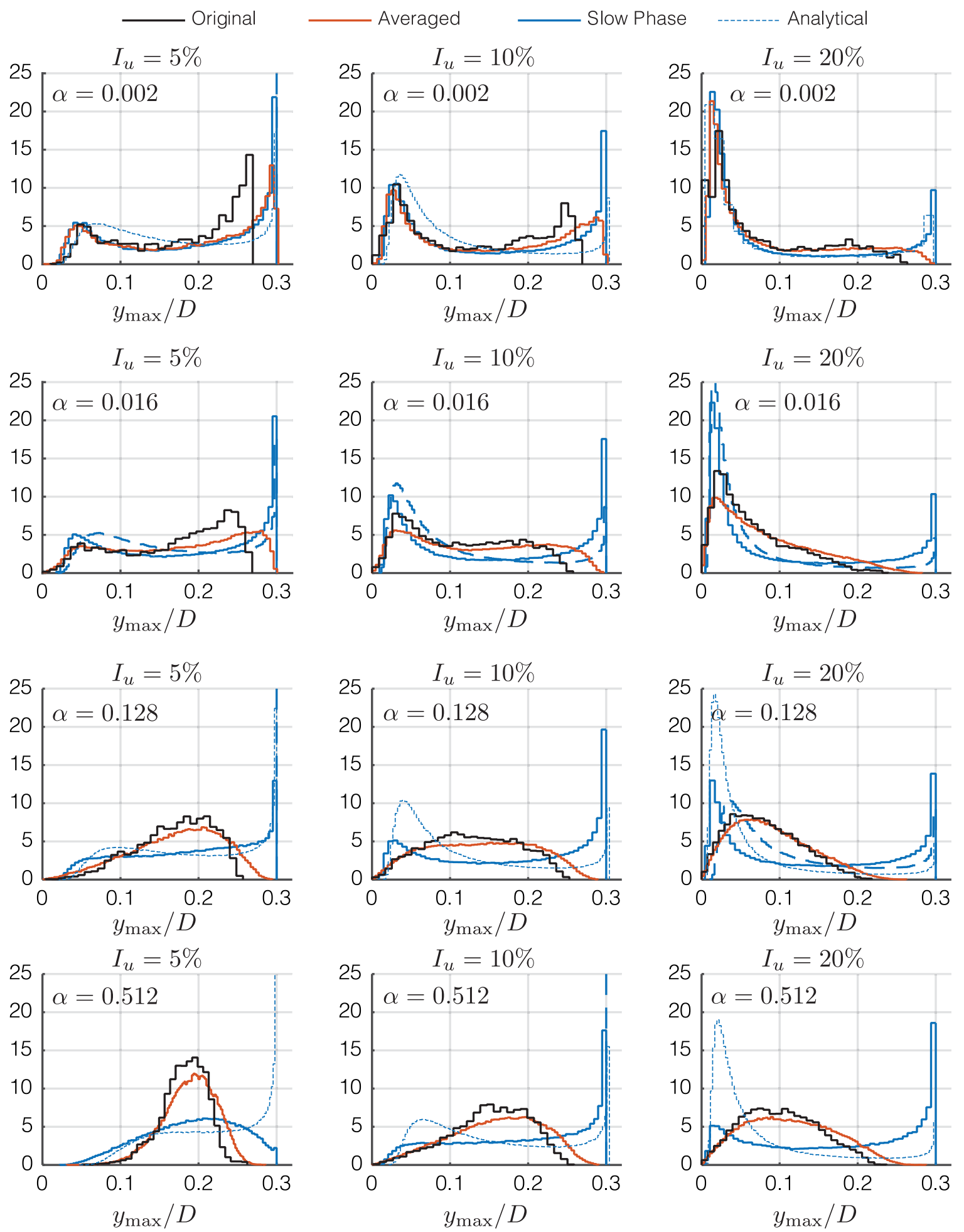

Fig. 15. Comparison of the probability density function of the envelope of the structural response $R_{y}$, obtained with the 4 models described in this paper: the original model (11), the averaged model (25), the slow phase model (41)-(42) and the analytical formulation corresponding to the small intensity asymptotic solution of the slow phase model. Numerical values : Data Set $1, \delta=2(\Omega=1.06), I_{u}$ variable, $\alpha$ variable. (For interpretation of the references to color in this figure legend, the reader is referred to the web version of this article.) 
is required to support the use, in turbulent conditions, of the slow phase on the unperturbed limit cycle (26). Indeed, when $\alpha \ll \xi$, the driving excitation of the averaged model (25) is slow, so is the response and $R_{y}^{\prime}(T)$ is negligible. This provides the optimal conditions for the slow phase model. As $\alpha$ becomes larger, the dynamics of the response envelope $R_{y}^{\prime}(T)$ becomes more important and the quality of the slow phase model worsens. Nevertheless it is mostly important that the simplified slow phase model and its approximate solution catch the right scaling, the right trends, and be accurate where necessary, i.e. where the turbulence precisely has an influence on the structural response.

The quality of the simple analytical model is confirmed for $\alpha=0.016$; the transition from lock-in to unlock condition is validated. For $\alpha=0.128$ and $\alpha=0.512$, the distribution of the response is less accurately predicted by the slow phase model and the proposed analytical formulation. This limitation only concerns large turbulence intensities; for both $\alpha=0.128$ and $\alpha=0.512$ and both considered values of $\delta$, the distribution of the response is well estimated in case of small turbulence intensity $I_{u}=5 \%$. For higher turbulence intensities, although the probability distribution of the response envelope is off, the slow phase model succeeds in qualitatively predicting the smooth transition from lock-in to unlock conditions.

The illustrations in Figs. 14 and 15 also indicate that the slow phase model predicts a dichotomic response envelope: either located near a perfect lock-in configuration, either located near an unlocked configuration. The transition from one to the other appears to be faster than in the original model. This is a consequence of the use of the memoryless transformation (42) of the slow phase model. As $\alpha$ gets larger, the dynamics of $R_{y}, R_{q}$ and $\psi$ become more intricate. This explains why the slow phase model is not able to reproduce bell shape distributions for $y_{\max } / D$ as $\alpha$ gets larger.

On a side note, we notice that the original (black) and averaged models (red) match perfectly in all parameter conditions represented in Figs. 14 and 15. This confirms the applicability of the well-known averaging technique and multiple scale analysis for this type of model.

\subsection{Influence of turbulence and mistuning}

Figs. 14 and 15 correspond to $\delta=0$ and $\delta=2$, but show the complete histogram of $y_{\max } / D$. A more detailed influence of the mistuning $\delta$ is given in Fig. 16, which shows the average envelope responses as a function of $\delta$. In that Figure, the thin continuous line in each subplot shows the lock-in response in smooth flow. It is the same reference, reported in all subplots, to serve an eye guide for comparison with the lock-in responses in turbulent conditions. The same four models as discussed before are compared. Again it is observed that the proposed analytical model and the whole chain of models having led to its establishment are fairly accurate when either the turbulence is a slow process $\alpha \ll 1$ (which is typically the case for wind turbulence), or when $I_{u} \ll 1$. In all circumstances, the proposed model provides a fair description of the influence of turbulence: when there is no influence, it is predicted so and when there is a reduction of VIV due to turbulence, the model is able to capture the main trends and orders of magnitude. It struggles a little more close to the limits of the lock-in domain, where the equivalent linear model fails to be as accurate as in perfect lock-in conditions.

\section{Conclusion}

The randomized wake-oscillator model is a possible candidate to phenomenologically model the influence of turbulence on vortex-induced vibrations. In the paper, we have presented an averaged version of this model. Then a slow phase model, based on a slow dynamics stochastic equation for the phase shift and a memoryless transformation for the response amplitude, has been derived. This model is simple enough to explain many features of the original model. In particular

- the phase variable is seen to play a central role in wake-oscillator models, the response amplitude certainly plays a secondary role;

- the dynamics of the two oscillators is better expressed by means of their (slow) envelope rather than (fast) oscillations; it is therefore recommended to refer to the statistics of the envelope rather than those of the oscillatory responses themselves, e.g. average of slow envelope response in lieu of the standard deviation of the fast dynamic response;

- as a consequence, while in the deterministic analysis of VIV, the response is sometimes represented by means of a root-mean-square amplitude, it is suggested to leave this concept behind and, instead, adopt a quantification which is related to the envelope (which is in fact straightforwardly managed with a $\sqrt{2}$ in the deterministic case);

- the averaging procedure has highlighted the important scalings in the problem; in particular, in forced conditions, the size of the lock-in region scales with $\varepsilon$ while, in free vibration conditions, it scales with the total damping ratio $\xi=\xi_{\mathrm{a}}+\xi_{\mathrm{s}}$ and the parameter $\mathcal{D}$ (see definition in (28));

- in both smooth and turbulent flows, the slow dynamics of the phase is governed by the dimensionless parameter $\mathcal{D}$ (see definition in (28));

- the analysis of the original model by means of perturbation methods has indicated that turbulence enters in the problem, at leading order, as a parametric modification in the wake equation; after averaging, it is seen that the turbulence just affects the phase equation and not the response envelope equations; 

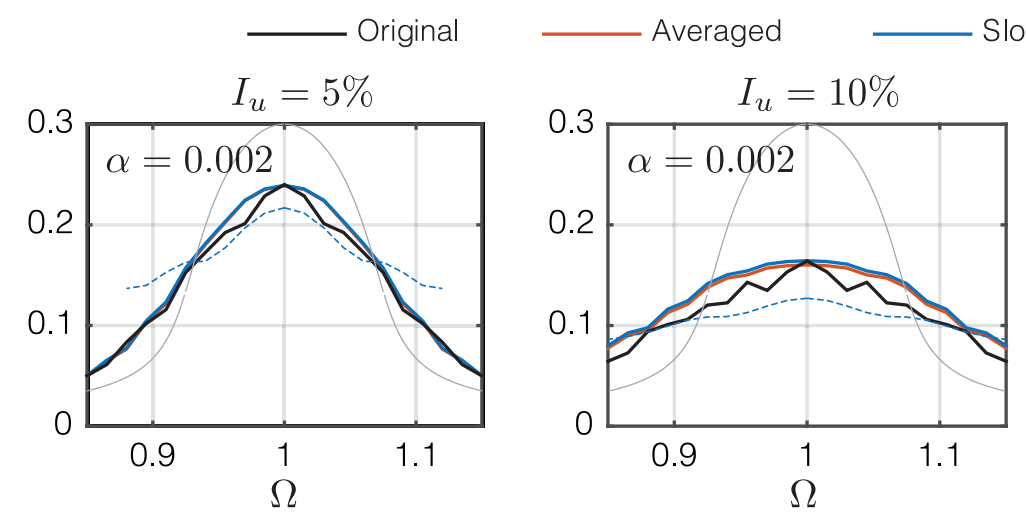

Slow Phase

Analytical
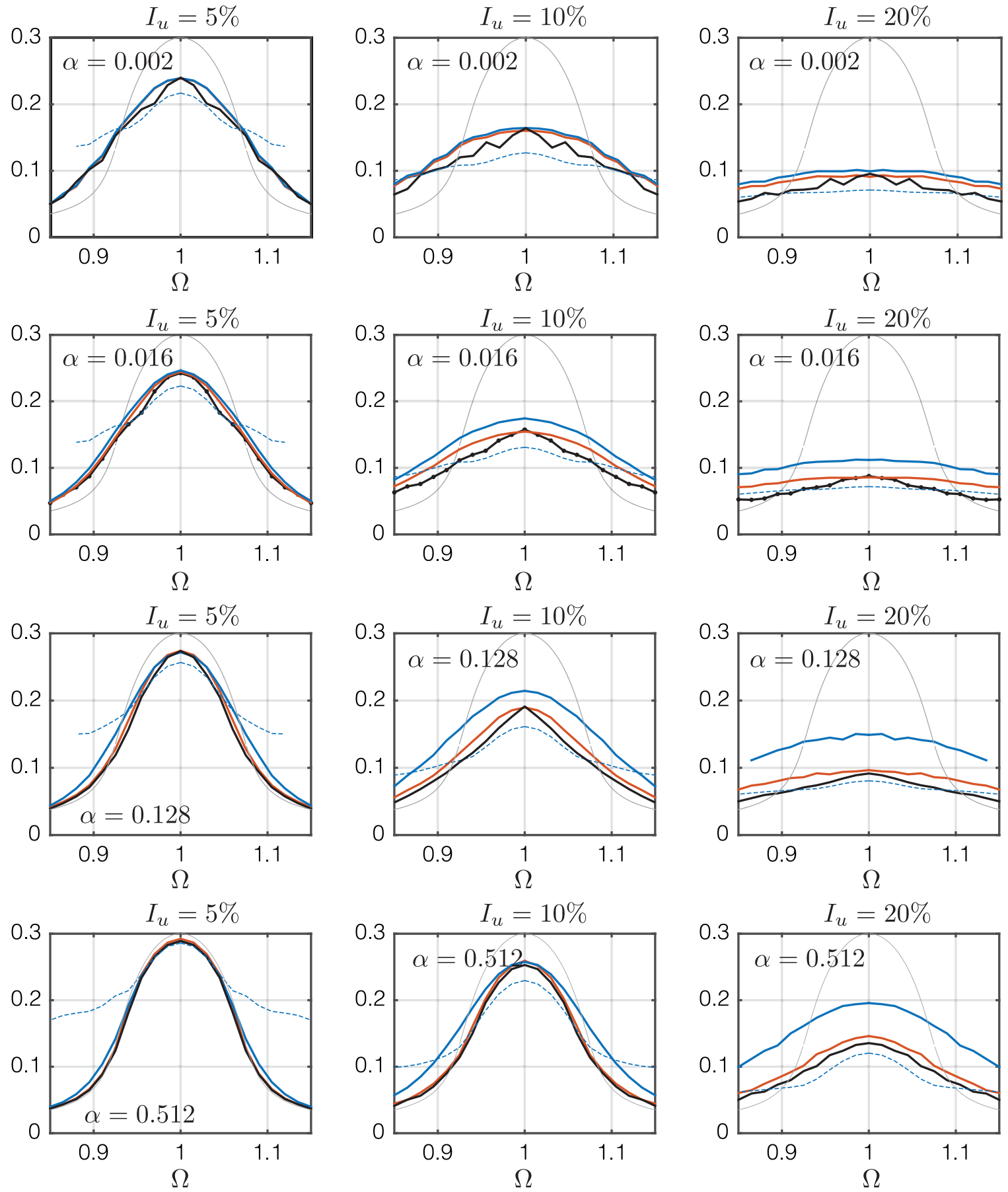

Fig. 16. Envelope of the structural response in the lock-in range as a function of turbulence intensity $I_{u}$ and turbulence characteristic frequency $\alpha$. The thin continuous line in all subplot shows the solution in smooth flow $\left(I_{u}=0\right)$. Numerical values : Data Set $1, I_{u} \in\{5 \%, 10 \%, 20 \%\}$, $\alpha \in\{0.002,0.016,0.128,0.512\}$.

- the slow phase model clearly establishes the intertwining relations between the timescales of the problem; in particular, the reduction of the VIV response due to turbulence is interpreted as an interaction of the slow timescale of the turbulence (related to $\alpha$ ) and the slow timescale of the coupled system (related to $\xi \mathcal{B}$ ). This explains for 
instance why a mathematical turbulence modeled as a white noise has virtually no influence on the deterministic response;

- as a corollary, this could also explain the controversy concerning the experimental evidences about the influence of turbulence on the structural response; indeed, not all experiments aiming at studying the influence of turbulence on the VIV response could highlight a significant influence; a possible explanation is that the frequency content (timescales) was not appropriately chosen to reveal such an influence (the appropriate frequency content depends on the damping ratio through $\alpha / \xi \mathcal{B}$, see (55)).

- the reduction of the VIV response due to turbulence is also interpreted through the increase of the standard deviation of the phase shift between the fluid and structural oscillator. By placing the phase shift in the center of the model, the influence of wind turbulence is assessed by means of the statistics of the phase.

- the well-known concept of phase slip and their accumulation (borrowed from other fields of sciences where random synchronization is studied) has also been touched to illustrate the increase of the standard deviation of the phase slip as the system is driven away from lock-in conditions;

In short, the ratio of the timescales of the turbulence and of the structural dynamics plays a significant role in the reduction of vibrations in the lock-in range: the slower the turbulence, the larger the reduction for a given turbulence intensity. Then of course the larger the turbulence intensity, the larger the reduction of VIV.

This work opens some perspectives on the topic. In particular, the use of multiple timescales in the analysis of VIV is encouraged since it focuses on the dynamics of the phase and of the envelope, which are central in the synchronization phenomenon. They are associated with slow dynamics only; based on similar computational resources, it is possible to obtain much more accurate results than when having to resolve the fast dynamics of the original problem. At last but not least, the developments presented in this paper are specific to the FDLB model but could be replicated for other wake-oscillator models available in the literature.

\section{CRediT authorship contribution statement}

Vincent Denoël: Conceptualization, Methodology, Software, Data curation, Visualization, Investigation, Supervision, Validation, Writing - review \& editing.

\section{Declaration of competing interest}

The authors declare that they have no known competing financial interests or personal relationships that could have appeared to influence the work reported in this paper.

\section{Appendix}

The appendix gathers the details of the multiple scale analysis of the governing equations. They are very similar to existing works in the literature (see e.g. Mannini, 2020) and are recalled here to make the notations consistent with the rest of the paper.

\section{A.1. Multiple scales analysis of the deterministic system: free vibration}

The dimensionless formulation of the governing equations is

$$
\begin{aligned}
\mathcal{Y}^{\prime \prime}+2 \xi \mathcal{Y}^{\prime}+\mathcal{Y} & =2 \varepsilon \mathcal{M}_{0} \mathcal{Q} \Omega^{2} \\
\mathcal{Q}^{\prime \prime}+\varepsilon \Omega\left(\mathcal{Q}^{2}-1\right) \mathcal{Q}^{\prime}+\Omega^{2} \mathcal{Q} & =2 \varepsilon \mathcal{A}_{0} \mathcal{Y}^{\prime \prime}
\end{aligned}
$$

The solution is sought in the form of the ansatz (23). The slow timescale $t^{\star} / \varepsilon$ and the slow time $T=t /\left(t^{\star} / \varepsilon\right)=\varepsilon \tau$ are introduced. Time derivatives with respect to time $\tau$ are replaced by partial derivatives as

$$
\frac{d}{d \tau}=\partial_{\tau}+\varepsilon \partial_{T} ; \quad \frac{d^{2}}{d \tau^{2}}=\partial_{\tau}^{2}+2 \varepsilon \partial_{\tau T}^{2}+\varepsilon^{2} \partial_{T}^{2} .
$$

Introducing the ansatz (23) into the governing equation and limiting the development to second order yields

$$
\begin{array}{r}
\left(\partial_{\tau}^{2}+2 \varepsilon \partial_{\tau T}^{2}+\varepsilon^{2} \partial_{T}^{2}\right)\left(\mathcal{Y}_{0}+\varepsilon \mathcal{Y}_{1}\right)+2 \xi\left(\partial_{\tau}+\varepsilon \partial_{T}\right)\left(\mathcal{Y}_{0}+\varepsilon \mathcal{Y}_{1}\right)+\left(\mathcal{Y}_{0}+\varepsilon \mathcal{Y}_{1}\right)=2 \varepsilon \mathcal{M}_{0} \mathcal{Q} \Omega^{2} \\
\left(\partial_{\tau}^{2}+2 \varepsilon \partial_{\tau T}^{2}+\varepsilon^{2} \partial_{T}^{2}\right)\left(\mathcal{Q}_{0}+\varepsilon \mathcal{Q}_{1}\right)+\varepsilon \Omega\left(\left(\mathcal{Q}_{0}+\varepsilon \mathcal{Q}_{1}\right)^{2}-1\right)\left(\partial_{\tau}+\varepsilon \partial_{T}\right)\left(\mathcal{Q}_{0}+\varepsilon \mathcal{Q}_{1}\right)+\Omega^{2}\left(\mathcal{Q}_{0}+\varepsilon \mathcal{Q}_{1}\right)=2 \varepsilon \mathcal{A}_{0} \mathcal{Y}^{\prime \prime}
\end{array}
$$

which then gives, limiting again to first two leading orders

$$
\begin{aligned}
\partial_{\tau}^{2} \mathcal{Y}_{0}+2 \varepsilon \partial_{\tau T}^{2} \mathcal{Y}_{0}+\varepsilon \partial_{\tau}^{2} \mathcal{Y}_{1}+2 \xi\left(\partial_{\tau} \mathcal{Y}_{0}+\varepsilon \partial_{T} \mathcal{Y}_{0}+\varepsilon \partial_{\tau} \mathcal{Y}_{1}\right)+\mathcal{Y}_{0}+\varepsilon \mathcal{Y}_{1} & =2 \varepsilon \mathcal{M}_{0} \mathcal{Q}_{0} \Omega^{2} \\
\partial_{\tau}^{2} \mathcal{Q}_{0}+2 \varepsilon \partial_{\tau T}^{2} \mathcal{Q}_{0}+\varepsilon \partial_{\tau}^{2} \mathcal{Q}_{1}+\varepsilon \Omega\left(\mathcal{Q}_{0}^{2}-1+2 \varepsilon \mathcal{Q}_{0} \mathcal{Q}_{1}\right) \partial_{\tau} \mathcal{Q}_{0}+\Omega^{2}\left(\mathcal{Q}_{0}+\varepsilon \mathcal{Q}_{1}\right) & =2 \varepsilon \mathcal{A}_{0} \partial_{\tau}^{2} \mathcal{Y}_{0} .
\end{aligned}
$$


We further assume that the structural damping is small, $\xi=\xi_{0} \varepsilon$, with $\xi_{0}$ of order 1 and $\varepsilon \ll 1$. We also focus on small detuning, $\Omega=1+\xi \delta=1+\xi_{0} \varepsilon \delta$, i.e. $\Omega^{2}=1+2 \xi_{0} \varepsilon \delta+\operatorname{ord}\left(\varepsilon^{2}\right)$, so that the set of governing equations becomes, after dropping second order terms,

$$
\begin{aligned}
\partial_{\tau}^{2} \mathcal{Y}_{0}+2 \varepsilon \partial_{\tau T}^{2} \mathcal{Y}_{0}+\varepsilon \partial_{\tau}^{2} \mathcal{Y}_{1}+2 \varepsilon \xi_{0} \partial_{\tau} \mathcal{Y}_{0}+\mathcal{Y}_{0}+\varepsilon \mathcal{Y}_{1} & =2 \varepsilon \mathcal{M}_{0} \mathcal{Q}_{0}\left(1+2 \varepsilon \xi_{0} \delta\right) \\
\partial_{\tau}^{2} \mathcal{Q}_{0}+2 \varepsilon \partial_{\tau T}^{2} \mathcal{Q}_{0}+\varepsilon \partial_{\tau}^{2} \mathcal{Q}_{1}+\varepsilon\left(\mathcal{Q}_{0}^{2}-1\right) \partial_{\tau} \mathcal{Q}_{0}+\left(1+2 \varepsilon \xi_{0} \delta\right)\left(\mathcal{Q}_{0}+\varepsilon \mathcal{Q}_{1}\right) & =2 \varepsilon \mathcal{A}_{0} \partial_{\tau}^{2} \mathcal{Y}_{0}
\end{aligned}
$$

Collecting the likewise powers of $\varepsilon$, it is possible to obtain the governing equations for $\mathcal{Y}_{0}$ and $\mathcal{Q}_{0}$, then $\mathcal{Y}_{1}$ and $\mathcal{Q}_{1}$, etc. In particular, at leading order

$$
\begin{aligned}
\partial_{\tau}^{2} \mathcal{Y}_{0}+\mathcal{Y}_{0} & =0 \\
\partial_{\tau}^{2} \mathcal{Q}_{0}+\mathcal{Q}_{0} & =0
\end{aligned}
$$

whose solution is

$$
\mathcal{Y}_{0}=R_{y}(T) \cos (\tau+\varphi(T)) \quad ; \quad \mathcal{Q}_{0}=R_{q}(T) \cos (\tau+\varphi(T)+\psi(T)) .
$$

At the next order, we have

$$
\begin{aligned}
\partial_{\tau}^{2} \mathcal{Y}_{1}+\mathcal{Y}_{1} & =2 \mathcal{M}_{0} \mathcal{Q}_{0}-2 \partial_{\tau T}^{2} \mathcal{Y}_{0}-2 \xi_{0} \partial_{\tau} \mathcal{Y}_{0} \\
\partial_{\tau}^{2} \mathcal{Q}_{1}+\mathcal{Q}_{1} & =2 \mathcal{A}_{0} \partial_{\tau}^{2} \mathcal{Y}_{0}-2 \partial_{\tau T}^{2} \mathcal{Q}_{0}-\left(\mathcal{Q}_{0}^{2}-1\right) \partial_{\tau} \mathcal{Q}_{0}-2 \xi_{0} \delta \mathcal{Q}_{0}
\end{aligned}
$$

1

2

The righthand sides can be developed in order to reveal the secular terms. Substitution of the expressions for $\mathcal{Y}_{0}$ and $\mathcal{Q}_{0}$ yields, after some development,

$$
\begin{aligned}
\partial_{\tau}^{2} \mathcal{Y}_{1}+\mathcal{Y}_{1}= & 2\left(\mathcal{M}_{0} R_{q} \cos \psi+R_{y} \varphi^{\prime}\right) \cos (\tau+\varphi)+2\left(-\mathcal{M}_{0} R_{q} \sin \psi+R_{y}^{\prime}+\xi_{0} R_{y}\right) \sin (\tau+\varphi) \\
\partial_{\tau}^{2} \mathcal{Q}_{1}+\mathcal{Q}_{1}= & 2\left(-\mathcal{A}_{0} R_{y} \cos \psi+R_{q}\left(\varphi^{\prime}+\psi^{\prime}\right)-\xi_{0} \delta R_{q}\right) \cos \tilde{\tau}+ \\
& \left(-2 \mathcal{A}_{0} R_{y} \sin \psi+2 R_{q}^{\prime}+\left(R_{q}^{2}-1\right) R_{q}-\frac{3}{4} R_{q}^{3}\right) \sin \tilde{\tau}+R_{q}^{3} \frac{\sin 3 \tilde{\tau}}{4} .
\end{aligned}
$$

where $\tilde{\tau}=\tau+\varphi(T)+\psi(T)$. It then appears that the slow magnitude and phases introduced in $\mathcal{Y}_{0}(\tau, T)$ and $\mathcal{Q}_{0}(\tau, T)$ need to satisfy

$$
\begin{aligned}
R_{q}^{\prime} & =\mathcal{A}_{0} R_{y} \sin \psi-\frac{1}{8} R_{q}^{3}+\frac{1}{2} R_{q} \\
R_{y}^{\prime} & =\mathcal{M}_{0} R_{q} \sin \psi-\xi_{0} R_{y} \\
\varphi^{\prime} & =-\mathcal{M}_{0} \frac{R_{q}}{R_{y}} \cos \psi \\
\varphi^{\prime}+\psi^{\prime} & =\mathcal{A}_{0} \frac{R_{y}}{R_{q}} \cos \psi+\xi_{0} \delta
\end{aligned}
$$

in order to not break the asymptoticness of the series. The last two equations can be combined in order to provide three first order equations governing the slow time evolution of $R_{q}(T), R_{y}(T)$ and $\psi(T)$, see (25). In particular, the slow time evolution of $\psi(T)$ plays a central role in (i) the synchronization of the system in case of external forcing (phase entrainment) or (ii) in the dispatching of the total energy between the fluid and the structure in the limit cycle, in case of autonomous system, without external forcing.

\section{A.2. Multiple scales analysis of the deterministic system: forced vibration}

In this section, the forced response of the system is derived. The dimensionless imposed amplitude of the body is chosen as $\mathcal{Y}_{0} \sin \tau$ and the bifurcation parameter of the formulation is $\Omega$, which is directly affected by the wind speed $U_{\infty}$. The dimensionless acceleration $\mathcal{Y}^{\prime \prime}$ is equal to $-\mathcal{Y}_{0} \sin \tau$ and the governing equations is

$$
\mathcal{Q}^{\prime \prime}+\varepsilon \Omega\left(\mathcal{Q}^{2}-1\right) \mathcal{Q}^{\prime}+\Omega^{2} \mathcal{Q}=-2 \varepsilon \mathcal{A}_{0} \mathcal{Y}_{0} \sin \tau
$$

The solution is sought in the form of the same ansatz as above and with the same two timescales. The only difference with the former development is that the mistuning is now defined by

$$
\Omega=1+\varepsilon \omega \Leftrightarrow \omega=\frac{\Omega-1}{\varepsilon}=\frac{f_{\text {shedding }}-f_{0}}{\varepsilon f_{0}} .
$$

This change of definition arises as the damping ratio $\xi$ does not appear in the governing equations as soon as the kinematics of the body are imposed. Limiting the developments to second order, it is natural to obtain a very similar expansion,

$$
\partial_{\tau}^{2} \mathcal{Q}_{0}+2 \varepsilon \partial_{\tau T}^{2} \mathcal{Q}_{0}+\varepsilon \partial_{\tau}^{2} \mathcal{Q}_{1}+\varepsilon\left(\mathcal{Q}_{0}^{2}-1\right) \partial_{\tau} \mathcal{Q}_{0}+(1+2 \varepsilon \omega)\left(\mathcal{Q}_{0}+\varepsilon \mathcal{Q}_{1}\right)=-2 \varepsilon \mathcal{A}_{0} \mathcal{Y}_{0} \sin \tau
$$


The solution at leading order yields

$$
\mathcal{Q}_{0}=R_{q}(T) \cos (\tau+\phi(T)) .
$$

At the next order, we have

$$
\partial_{\tau}^{2} \mathcal{Q}_{1}+\mathcal{Q}_{1}=-2 \mathcal{A}_{0} \mathcal{Y}_{0} \sin \tau-2 \partial_{\tau T}^{2} \mathcal{Q}_{0}-\left(\mathcal{Q}_{0}^{2}-1\right) \partial_{\tau} \mathcal{Q}_{0}-2 \omega \mathcal{Q}_{0} \quad .
$$

Working out again the righthand side in the same manner as before, the secular terms can be identified,

$$
\begin{aligned}
\partial_{\tau}^{2} \mathcal{Q}_{1}+\mathcal{Q}_{1}= & -2 \mathcal{A}_{0} \mathcal{Y}_{0} \sin \tau+2 R_{q}^{\prime} \sin (\tau+\phi)+2 R_{q} \phi^{\prime} \cos (\tau+\phi) \\
& +\left(R_{q}^{2}-1\right) R_{q} \sin (\tau+\phi)-R_{q}^{3} \sin ^{3}(\tau+\phi)-2 \omega R_{q} \cos (\tau+\phi) \\
= & {\left[-2 \mathcal{A}_{0} \mathcal{Y}_{0} \cos \phi+2 R_{q}^{\prime}-R_{q}+\frac{1}{4} R_{q}^{3}\right] \sin (\tau+\phi) } \\
& +\left[2 \mathcal{A}_{0} \mathcal{Y}_{0} \sin \phi+2 R_{q} \phi^{\prime}-2 \omega R_{q}\right] \cos (\tau+\phi)+\frac{R_{q}^{3}}{4} \sin 3(\tau+\phi),
\end{aligned}
$$

and the secularity conditions, used to determine the slow amplitude and the slow time shift in $\mathcal{Q}_{0}$, are

$$
\begin{aligned}
R_{q}^{\prime} & =\mathcal{A}_{0} \mathcal{Y}_{0} \cos \phi-\frac{1}{8} R_{q}^{3}+\frac{1}{2} R_{q} \\
\phi^{\prime} & =\omega-\frac{\mathcal{A}_{0} \mathcal{Y}_{0}}{R_{q}} \sin \phi .
\end{aligned}
$$

This set of equations is known to exhibit different solutions depending on the ratio $\mathcal{A}_{0} \mathcal{Y}_{0} / \omega$ (Glendinning, 1994). The fixed points are obtained by canceling the righthand sides in the two equations above. Combining with the trigonometry identity, it is possible to obtain

$$
\left(\mathcal{A}_{0} \mathcal{Y}_{0}\right)^{2}=\frac{1}{4} R_{q}^{2}\left(\frac{1}{4} R_{q}^{2}-1\right)^{2}+R_{q}^{2} \omega^{2} .
$$

Following the same arguments as in Glendinning (1994) it is possible to prove that:

- if $\mathcal{A}_{0} \mathcal{Y}_{0} \leq 2 \sqrt{3} / 9 \simeq 0.385$, a stable fixed point exists if and only if there are 3 real roots, i.e.

$$
\left(2-27\left(\mathcal{A}_{0} \mathcal{Y}_{0}\right)^{2}+72 \omega^{2}\right)^{2} \geq 4\left(1-12 \omega^{2}\right)^{3},
$$

in which case the largest root is the stable fixed point. Solving this inequality for $\omega$ yields, for small $\mathcal{A}_{0} \mathcal{Y}_{0}$,

$$
|\omega| \leq \frac{\mathcal{A}_{0} \mathcal{Y}_{0}}{2} \sqrt{\frac{\left(\mathcal{A}_{0} \mathcal{Y}_{0}\right)^{2}}{4}+1}
$$

This means that frequency locking (synchronization) is observed as long as the mistuning is not too large.

- if $2 \sqrt{3} / 9 \leq \mathcal{A}_{0} \mathcal{Y}_{0} \leq 2 \sqrt{6} / 9 \simeq 0.544$, as $\omega$ increases from zero to $+\infty$, the equation in $R_{q}$ has just one root, then three, then one again. The stable point corresponding to the largest root looses stability at the transition from 3 roots to one root. Synchronization takes place in similar conditions as above.

- if $\mathcal{A}_{0} \mathcal{Y}_{0} \geq 2 \sqrt{6} / 9$, the polynomial in $R_{q}$ has only one root, no matter the value of $\omega$. This root is again stable as long as $|\omega|$ is smaller than a stability limit. Stability is lost when the isocline associated with $R_{q}^{\prime}=0$ has a vertical tangent in the $\left(R_{q} \cos \phi, R_{q} \sin \phi\right)$-plane. This condition reads

$$
|\omega| \leq \frac{1}{4} \sqrt{8\left(\mathcal{A}_{0} \mathcal{Y}_{0}\right)^{2}-1}
$$

These stability limits on $|\omega|$ correspond to the frequency locking (synchronization) range. By noticing that $2 \mathcal{A}_{0} \mathcal{Y}_{0}=$ $\frac{2 \mathcal{A}_{0}}{\varepsilon} \mathcal{Y}_{0} \varepsilon=\frac{A}{\varepsilon} \frac{y}{D}$, they can be expressed with the original parameters of the problem.

\section{References}

Aswathy, M.S., Sarkar, Sunetra, 2019. Effect of stochastic parametric noise on vortex induced vibrations. Int. J. Mech. Sci. 153-154, 103-118. Benaroya, Haym, Lepore, JA, 1983. Statistical flow-oscillator modeling of vortex-shedding. J. Sound Vib. 86 (2), $159-179$.

Bender, Carl M, Orszag, Steven A, 2013. Advanced Mathematical Methods for Scientists and Engineers I: Asymptotic Methods and Perturbation Theory. Springer Science \& Business Media.

Birkhoff, Garrett, 1953. Formation of vortex streets. J. Appl. Phys. 24 (1), 98-103.

Blackburn, HM, Melbourne, WH, 1996. The effect of free-stream turbulence on sectional lift forces on a circular cylinder. J. Fluid Mech. 306, 267-292.

Canor, Thomas, Denoël, Vincent, 2013. Transient fokker-planck-kolmogorov equation solved with smoothed particle hydrodynamics method. Internat. J. Numer. Methods Engrg. 94 (6), 535-553.

Cao, Shuyang, Cao, Jinxin, 2017. Toward better understanding of turbulence effects on bridge aerodynamics. Front. Built Environ. 3, 72.

Chang, JS, Cooper, G, 1970. A practical difference scheme for fokker-planck equations. J. Comput. Phys. 6 (1), 1-16. 
Cremona, Christian, et al., 2002. Comportement au vent des ponts.

Daly, AF, 1986. Evaluation of methods of predicting the across-wind response of chimneys. CICIND Rep. 2 (1).

Denoël, Vincent, 2015. Multiple timescale spectral analysis. Probab. Eng. Mech. 39, 69-86.

Denoël, Vincent, Andrianne, Thomas, 2017. Real-scale observations of vortex induced vibrations of stay-cables in the boundary layer. Procedia Eng. 199, 3109-3114, X International Conference on Structural Dynamics, EURODYN 2017.

Denoël, Vincent, Carassale, Luigi, 2015. Response of an oscillator to a random quadratic velocity-feedback loading. J. Wind Eng. Ind. Aerodyn. 147, 330-344.

Dyrbye, Claës, Hansen, Svend Ole, 1996. Wind Loads on Structures. Wiley.

Facchinetti, Matteo Luca, De Langre, Emmanuel, Biolley, Francis, 2004. Coupling of structure and wake oscillators in vortex-induced vibrations. J. Fluids Struct. 19 (2), 123-140.

Farshidianfar, A, Zanganeh, Hossein, 2010. A modified wake oscillator model for vortex-induced vibration of circular cylinders for a wide range of mass-damping ratio. J. Fluids Struct. 26, 430-441.

Glendinning, Paul, 1994. Stability, Instability and Chaos: an Introduction to the Theory of Nonlinear Differential Equations, Vol. 11. Cambridge university press.

Goswami, Indranil, Scanlan, Robert H, Jones, Nicholas P, 1993. Vortex-induced vibration of circular cylinders. i: experimental data. J. Eng. Mech. 119 (11), 2270-2287.

Griffin, OM, Koopmann, GH, 1977. The vortex-excited lift and reaction forces on resonantly vibrating cylinders. J. Sound Vib. 54 (3), $435-448$.

Gu, Rencai, Xu, Yong, Hao, Mengli, Yang, Zhiqiang, 2011. Stochastic bifurcations in duffing-van der pol oscillator with Lévy stable noise. Acta Phys. Sin. 60 .

Guckenheimer, John, 1975. Isochrons and phaseless sets. J. Math. Biol. 1 (3), 259-273.

Guilmineau, E., Queutey, P., 2004. Numerical simulation of vortex-induced vibration of a circular cylinder with low mass-damping in a turbulent flow. J. Fluids Struct. 19 (4), 449-466.

Hansen, Svend Ole, 2007. Vortex-induced vibrations of structures. In: Structural Engineers World Congress. pp. 2-7.

Hartlen, Ronald T, Currie, Iain G, 1970. Lift-oscillator model of vortex-induced vibration. J. Eng. Mech. Div. 96 (5), 577-591.

Howell, JF, Novak, M, 1980. Vortex shedding from circular cylinders in turbulent flow. In: Wind Engineering. Elsevier, pp. 619-629.

Kawai, H, 1992. Vortex induced vibration of tall buildings. J. Wind Eng. Ind. Aerodyn. 41 (1), 117-128.

Komatsu, S, Kobayashi, H, 1980. Vortex-induced oscillation of bluff cylinders. J. Wind Eng. Ind. Aerodyn. 6 (3-4), 335-362.

Krenk, Steen, Nielsen, Søren RK, 1999. Energy balanced double oscillator model for vortex-induced vibrations. J. Eng. Mech. 125 (3), $263-271$.

Larsen, Allan, 1995. A generalized model for assessment of vortex-induced vibrations of flexible structures. J. Wind Eng. Ind. Aerodyn. 57 (2), 281-294, Proceedings of the First IAWE European and African Regional Conference.

Le, Thai-Hoa, Caracoglia, Luca, 2015. Modeling vortex-shedding effects for the stochastic response of tall buildings in non-synoptic winds. J. Fluids Struct. 61, 461-491.

Leung, H.K., 1995. Stochastic transient of a noisy van der pol oscillator. Physica A 221 (1), 340-347, Proceedings of the Second IUPAP Topical Conference and the Third Taipei International Symposium on Statistical Physics.

Li, Lingyao, 2019. Analysis of uncertainty influence on the probabilistic evaluation of vortex-induced vibration response of a self-anchored suspension bridge. KSCE J. Civ. Eng. 23 (11), 4790-4799.

Lupi, Francesca, Niemann, Hans-Jürgen, Höffer, Rüdiger, 2018. Aerodynamic damping model in vortex-induced vibrations for wind engineering applications. J. Wind Eng. Ind. Aerodyn. 174, 281-295.

Mannini, Claudio, 2020. Asymptotic analysis of a dynamical system for vortex-induced vibration and galloping. In: Nonlinear Dynamics of Structures, Systems and Devices. Springer, pp. 389-397.

Marra, Antonino M, Mannini, Claudio, Bartoli, Gianni, 2011. Van der pol-type equation for modeling vortex-induced oscillations of bridge decks. J. Wind Eng. Ind. Aerodyn. 99 (6-7), 776-785.

Marra, Antonino Maria, Mannini, Claudio, Bartoli, Gianni, 2015. Measurements and improved model of vortex-induced vibration for an elongated rectangular cylinder. J. Wind Eng. Ind. Aerodyn. 147, 358-367.

Matlab, 2012. Matlab. The MathWorks Inc., Natick, Massachusetts, USA, Release 2012b.

Matsumoto, Masaru, Yagi, Tomomi, Shigemura, Yoshinori, Tsushima, Daisuke, 2001. Vortex-induced cable vibration of cable-stayed bridges at high reduced wind velocity. J. Wind Eng. Ind. Aerodyn. 89 (7), 633-647, 10th International Conference on Wind Engineering.

Nayfeh, Ali H, Mook, Dean T, 2008. Nonlinear Oscillations. John Wiley \& Sons.

Nguyen, Dinh Tung, Hargreaves, David M, Owen, John S, 2018. Vortex-induced vibration of a 5: 1 rectangular cylinder: a comparison of wind tunnel sectional model tests and computational simulations. J. Wind Eng. Ind. Aerodyn. 175, 1-16.

Nielsen, Søren R.K., Krenk, S., 1997. Stochastic Response of Energy Balanced Model for Vortex-Induced Vibration. Dept. of Building Technology and Structural Engineering.

Païdoussis, Michael P, Price, Stuart J, De Langre, Emmanuel, 2010. Fluid-Structure Interactions: Cross-Flow-Induced Instabilities. Cambridge University Press.

Papoulis, Athanasios, Pillai, S Unnikrishna, 2002. Probability, Random Variables, and Stochastic Processes. Tata McGraw-Hill Education.

Pasto, S., 2008. Vortex-induced vibrations of a circular cylinder in laminar and turbulent flows. J. Fluids Struct. 24 (7), 977-993.

Pikovsky, Arkady, Kurths, Jurgen, Rosenblum, Michael, Kurths, Jürgen, 2003. Synchronization: A Universal Concept in Nonlinear Sciences, Vol. 12. Cambridge university press.

Pritchard, BN, 1984. Steel chimney oscillations: a comparative study of their reported performance versus predictions using existing design techniques. Eng. Struct. 6 (4), 315-323.

Risken, Hannes, 1996. Fokker-planck equation. In: The Fokker-Planck Equation. Springer, pp. 63-95.

Ruscheweyh, H, Sedlacek, G, 1988. Crosswind vibrations of steel stacks.-critical comparison between some recently proposed codes. J. Wind Eng. Ind. Aerodyn. 30 (1-3), 173-183.

Sarwar, Muhammad Waheed, Ishihara, Takeshi, 2010. Numerical study on suppression of vortex-induced vibrations of box girder bridge section by aerodynamic countermeasures. J. Wind Eng. Ind. Aerodyn. 98 (12), 701-711.

Scruton, C, 1981. An Introduction to Wind Effects on Structures, Vol. 3. Oxford University Press Oxford.

Shoshani, O., 2018. Deterministic and stochastic analyses of the lock-in phenomenon in vortex-induced vibrations. J. Sound Vib. 434, 17-27.

Simiu, Emil, Scanlan, Robert H, 1996. Wind Effects on Structures: Fundamentals and Applications to Design. Wiley New York.

Skop, RA, Balasubramanian, S, 1997. A new twist on an old model for vortex-excited vibrations. J. Fluids Struct. 11 (4), 395-412.

So, R.M.C., Wang, X.Q., Xie, W.-C., Zhu, J., 2008. Free-stream turbulence effects on vortex-induced vibration and flow-induced force of an elastic cylinder. J. Fluids Struct. 24 (4), 481-495.

Soti, Atul Kumar, Zhao, Jisheng, Thompson, Mark C, Sheridan, John, Bhardwaj, Rajneesh, 2018. Damping effects on vortex-induced vibration of a circular cylinder and implications for power extraction. J. Fluids Struct. 81, 289-308.

Spencer, BF, Bergman, LA, 1993. On the numerical solution of the fokker-Planck equation for nonlinear stochastic systems. Nonlinear Dynam. 4 (4), $357-372$. 
Standard, European, 1991. 1991-1-4: 2005. Eurocode 1: Actions on Structures. Part 1-4: General Actions-Wind Actions. European Committee for Standardization (CEN) Brussels.

Tagliaferri, Francesca, Srinil, Narakorn, 2017. Quantifying Uncertainties in Phenomenological Model of Two-Dimensional VIV Using Multivariate Monte Carlo Simulations. In: International Conference on Offshore Mechanics and Arctic Engineering, Volume 2: Prof. Carl Martin Larsen and Dr. Owen Oakley Honoring Symposia on CFD and VIV, V002T08A026.

TAMURA, Y, Matsui, G, 1980. Wake-oscillator model of vortex-induced oscillation of circular cylinder. In: Wind Engineering. Elsevier, pp. 1085-1094.

Trush, Arsenii, Pospíšil, Stanislav, Kuznetsov, Sergey, Kozmar, Hrvoje, 2017. Wind-tunnel experiments on vortex-induced vibration of rough bridge cables. J. Bridge Eng. 22 (10), 06017001.

Ulveseter, J.V., Thorsen, M.J., Saevik, S., Larsen, C.M., 2017. Stochastic modelling of cross-flow vortex-induced vibrations. Mar. Struct. 56, 260-280.

Vickery, BJ, 1995. The response of chimneys and tower-like structures to wind loading. In: A State of the Art in Wind Engrg., Ninth Int. Conf. on Wind Engrg.. pp. 205-233.

Vickery, Barry J, 1998. Wind loads and design criteria for chimneys. Wind Effects Buidings Struct..

Vickery, BJ, Basu, RI, 1983. Across-wind vibrations of structures of circular cross-section. part i. development of a mathematical model for two-dimensional conditions. J. Wind Eng. Ind. Aerodyn. 12 (1), 49-73.

Vickery, Barry J, Clark, Arthur W, 1972. Lift or across-wind response to tapered stacks. J. Struct. Div. 98 (1), 1-20.

Williamson, Charles HK, Roshko, Anatol, 1988. Vortex formation in the wake of an oscillating cylinder. J. Fluids Struct. 2 (4), 355-381.

Winfree, Arthur T, 2001. The Geometry of Biological Time, Vol. 12. Springer Science \& Business Media.

Wu, Teng, Kareem, Ahsan, 2013. Vortex-induced vibration of bridge decks: Volterra series-based model. J. Eng. Mech. 139 (12), $1831-1843$.

Zeinoddini, M, Bakhtiari, A, Gharebaghi, S Asil, 2018. Towards an understanding of the marine fouling effects on viv of circular cylinders: a probe into the chaotic features. Nonlinear Dynam. 94 (1), 575-595. 